Andrea Schneiker

\title{
Epistemische Gemeinschaften und transnationale governance
}

\author{
Humanitäre Sicherheitsexperten als Motor für Wandel
}

Seit einigen Jahren werden humanitäre Nichtregierungsorganisationen (NGOs) zunehmend Opfer gewaltsamer Übergriffe. Dies stellt die Identität humanitärer NGOs in Frage, denn gemäß ihrem Selbstverständnis gewähren ihnen ihre Neutralität, Unabhängigkeit und Unparteilichkeit automatisch Schutz. Entsprechend schwer fällt es NGOs, ihr Sicherheitsverständnis zu überdenken. Umso erstaunlicher ist es, dass zahlreiche NGOs inzwischen ein internes Sicherheitsmanagement etabliert haben und sich untereinander vermehrt über Sicherheitsfragen austauschen. Auf Basis 27 leitfadengestützter qualitativer Interviews wird diese Entwicklung mit dem Engagement einer epistemischen Gemeinschaft humanitärer Sicherheitsexperten erklärt. Im Unterschied zum herkömmlichen Verständnis epistemischer Gemeinschaften setzt sich die hier untersuchte weder ausschließlich noch vorrangig aus Wissenschaftlern zusammen, sondern schließt Individuen aus unterschiedlichen Sektoren ein, die neben einer fachlichen Expertise auch bestimmte Werte teilen. Das daraus entstehende professionelle Selbstverständnis hält die epistemische Gemeinschaft nach innen zusammen und verschafft ihr nach außen legitime Autorität, so dass die von ihr gesetzten Standards nicht nur von zahlreichen NGOs, sondern auch von Regierungen anerkannt werden.

\section{Einleitung ${ }^{1}$}

Wie können wir die Entstehung transnationaler governance-Strukturen erklären, insbesondere in Fällen, in denen es seitens der betroffenen Akteure Widerstand gegen Wandel gibt? Der vorliegende Aufsatz erklärt dies mit der Arbeit einer Gruppe engagierter Experten, die als epistemische Gemeinschaft verstanden werden kann. Dabei leistet der Aufsatz einen Beitrag zur aktuellen Debatte über die Neubestimmung des Konzepts der epistemischen Gemeinschaft. Er erläutert, wie epistemische Gemeinschaften entstehen, welche Faktoren für den internen Zusammenhalt einer epistemischen Gemeinschaft entscheidend sind und wie eine epistemische Gemeinschaft zu strukturellem Wandel innerhalb einzelner Organisationen und zwischen Organisationen beiträgt. Das Politikfeld der humanitären Hilfe ist besonders geeignet, um diese Funktion epistemischer Gemeinschaften zu erörtern, da humanitäre

1 Für wertvolle Hinweise und Kommentare möchte ich insbesondere Jutta Joachim, Romina Ranke, Natalia Dalmer, Jens Taken und den drei anonymen Gutachter innen der ZIB danken. Des Weiteren danke ich Pia Honerath, Wiebke Pielhop, Debra Noe und Henriette Lange für ihre Unterstützung. 
Hilfe in den letzten Jahren aufgrund zahlreicher Entwicklungen immer wieder in Frage gestellt wird (Barnett/Weiss 2008a).

Humanitäre Hilfe wird heutzutage häufig in Kontexten bewaffneter Konflikte geleistet und in diesem Zusammenhang hat sich in den letzten Jahren in einigen Regionen der Welt die Sicherheitslage für die Arbeit humanitärer Organisationen verschlechtert (Stoddard et al. 2006; 2009: 1-4; 2011: 1; Wille/Fast 2013a: 1). Im Jahr 2011 erreichte dieser Trend seinen bisherigen Höhepunkt, als insgesamt 308 Mitarbeiter von Hilfsorganisationen Opfer gewaltsamer Übergriffe wurden (Humanitarian Outcomes 2012: 1). Diese Entwicklung stellt nicht nur jede einzelne Organisation vor Herausforderungen, sondern betrifft die »humanitäre Community « ${ }^{2}$ als Ganzes. Dieser Aufsatz fokussiert dabei humanitäre nicht-profitorientierte Nichtregierungsorganisationen (NGOs), da diese ganz besonders betroffen sind. Jene gingen bis vor kurzem davon aus, dass eine an den Prinzipien Neutralität, Unabhängigkeit und Unparteilichkeit ausgerichtete Hilfe Bedürftiger automatisch die Akzeptanz ihrer Arbeit durch die lokale Bevölkerung zur Folge hat und dadurch ihr eigener Schutz gewährleistet ist (Fast/O’Neill 2010: 4-6; Stoddard/Harmer 2010: 3). Dies ist jedoch angesichts der teilweise gezielten Angriffe auf NGOs heute nicht mehr der Fall. Somit stellt die Unsicherheit nicht nur die Durchführung humanitärer Programme, sondern auch die grundlegenden Prinzipien humanitärer Hilfe und damit die Identität humanitärer NGOs in Frage (Vaughn 2009: 264). Diese wird auch durch andere Entwicklungen wie zum Beispiel die Politisierung, Militarisierung und Kommerzialisierung humanitärer Hilfe herausgefordert (siehe z.B. Eberwein 2004: 2-6, 33-38; Gebauer 2002: 57-59; Spearin 2008: 347). Entsprechend sind humanitäre NGOs doppelt bedroht: einerseits von einer physischen Unsicherheit und andererseits von einer »ontologischen Unsicherheit« (Barnett/Weiss 2008b: 5).

Daher können sich humanitäre NGOs nicht mehr "passiv« darauf verlassen, dass sie auf Basis ihrer humanitären Arbeit automatisch Schutz vor Angriffen genießen, sondern sie sind gefordert, sich »aktiv« mit (ihrer) Sicherheit auseinanderzusetzen (Fast/O’Neill 2010: 5; Stoddard/Harmer 2010: 3). Dies betrifft einerseits ihre eigenen organisationsinternen Strukturen, Prozesse und Praktiken, aber andererseits auch den Austausch untereinander, denn das Handeln einer NGO kann Auswirkungen auf die Arbeit anderer NGOs haben und die Weitergabe sicherheitsrelevanter

2 Der Begriff der humanitären Community ist umstritten, weil er eine gewisse Homogenität der humanitären Akteure hinsichtlich ihrer Motivationen, Ziele und Vorgehensweisen suggeriert (Van Brabant 2010: 11). In der Realität unterscheiden sich Hilfsakteure aber teilweise erheblich mit Blick auf die genannten Faktoren und stehen untereinander im Wettbewerb, vor allem um Gelder (Van Brabant 2010: 11; Cooley/Ron 2002: 9). Trotz dieser Unterschiede soll hier von der humanitären Community gesprochen werden, um die Gesamtheit aller Akteure, die an der Durchführung humanitärer Hilfe beteiligt sind, zu erfassen. Dies schließt auch profitorientierte Akteure und zwischenstaatliche Organisationen wie die Vereinten Nationen mit ein. Sofern nur nicht-profitorientierte Nichtregierungsorganisationen (NGOs) gemeint sind, ist von der NGO-Community die Rede. Um den Eindruck zu vermeiden, die bestehenden Unterschiede übergehen zu wollen, werden die jeweiligen Begriffe in Anführungszeichen gesetzt. 
Informationen kann Leben retten. ${ }^{3}$ Entsprechende Veränderungen sind jedoch keine reinen Anpassungsprozesse, sondern fordern die grundlegenden Prinzipien humanitärer NGOs heraus (Argyris/Schön 2007: 18; Ebrahim 2005: 109; Van Brabant 2001a: 184). Daher existieren innerhalb der einzelnen NGOs zahlreiche Widerstände sowohl für die Etablierung eines internen Sicherheitsmanagements als auch die Kooperation mit anderen NGOs. Entsprechende Prozesse verlaufen folglich nicht konfliktfrei. Dennoch verfügen immer mehr NGOs über eine Sicherheitspolitik und darauf aufbauende Verfahren, um zum Beispiel die Sicherheitslage in einem Gebiet zu bestimmen, haben einen eigenen Sicherheitsmanager und bieten ihren Mitarbeitern Sicherheitstrainings an (Stoddard/Harmer 2010: 3; Van Brabant 2010: 5). Des Weiteren ist eine Zunahme institutionalisierter Sicherheitskooperationen von NGOs zu verzeichnen (Bickley 2006: 1, 5-16; Stoddard/Harmer 2010: 14-17).

Es liegt nahe, eine Erklärung für diese Entwicklungen in der schlechteren Sicherheitslage in den 2000er-Jahren im Vergleich zu den 1990er-Jahren zu suchen. Zwischen 1997 und 2008 ist die absolute Zahl gewaltsamer Übergriffe auf Mitarbeiter von Hilfsorganisationen um den Faktor fünf angestiegen und der prozentuale Anteil an Mitarbeitern von Hilfsorganisationen, die Opfer gewaltsamer Übergriffe wurden, hat sich verdoppelt (Stoddard et al. 2009: 2-3). ${ }^{4}$ Eine weitere mögliche Erklärung ist, dass humanitäre NGOs aus Sorge vor rechtlichen Folgen Sicherheitsmaßnahmen etablieren, um im Unglücksfall nicht von ihren Mitarbeitern verklagt zu werden. Doch nicht in allen Ländern fordern Geber oder Versicherungen entsprechende Standards ein und insgesamt verbleiben zahlreiche rechtliche Grauzonen (Van Brabant 2010: 6). Allgemein verfügen nicht alle humanitären NGOs über ein eigenes Sicherheitsmanagement (Gaul et al. 2006: 32; InterAction 2013) und einige NGOs sind nicht von der Relevanz von Kooperationen überzeugt (Micheni 2006: 365) oder nehmen aus anderen Gründen nicht daran teil. ${ }^{5}$ Somit können weder die Sicherheitslage noch rechtliche Rahmenbedingungen alleine erklären, dass zahlreiche humanitäre NGOs trotz Widerständen seit einigen Jahren ihren Umgang mit Sicherheit überdenken und verändern.

Der vorliegende Aufsatz erklärt diese Entwicklung mit den Aktivitäten einer epistemischen Gemeinschaft humanitärer Sicherheitsexperten, die Ende der 1990erJahre entstanden ist. Diese besteht aus einigen für Sicherheit innerhalb humanitärer

3 Bickley 2006: 1; Humanitarian Practice Network 2010b: 17; Micheni 2006: 365; Micheni/ Kuhanendran 2010: 8; Mujawar 2009: 11.

4 Nach einer anderen Quelle hat sich die Zahl der Opfer von Gewalt mit Todesfolge zwischen 2001 und 2011 verdreifacht (Wille/Fast 2013a: 1). Für eine differenzierte Darstellung darüber, welche Personengruppen und Länder mehr und welche weniger stark betroffen sind, siehe Wille/Fast (2013a: 1, 4-5; 2013b: 2, 9-23); Stoddard et al. (2011: 1, 3-9; 2012: 1, 3-4). Die Gründe für die zunehmende Unsicherheit sind umstritten. Für eine Diskussion siehe Fast (2010).

5 Interview mit einem NGO-Sicherheitsexperten, 20.11.2009. 
NGOs zuständigen Personen, so genannten Sicherheitsmanagern, ${ }^{6}$ Wissenschaftlern sowie freiberuflichen oder angestellten Beratern und Analysten. Das Ziel dieser epistemischen Gemeinschaft ist die Professionalisierung des Sicherheitsmanagements humanitärer NGOs entsprechend bestimmter Standards. Bezüglich des organisationsinternen Sicherheitsmanagements der einzelnen NGOs ist damit die Etablierung eines Systems gemeint, das eine Sicherheitspolitik und konkrete Verfahren zu deren Umsetzung im Rahmen bestimmter Strukturen umfasst (Van Brabant 2001b: 25-28). Mit Blick auf die Beziehungen zwischen NGOs ist damit die Etablierung von Strukturen zur Kooperation zwischen den NGOs gemeint, vor allem im Sinne eines regelmäßigen Informationsaustauschs.

Die epistemische Gemeinschaft verfügt über ausreichend professionelle Expertise sowie Autorität (Haas 1992a: 3), um im Bereich der humanitären Hilfe Widerstände gegen Veränderungen zu überwinden und Standards zu setzen, die von einer signifikanten Menge an humanitären NGOs anerkannt werden und für diese handlungsleitend sind. Dabei kann die epistemische Gemeinschaft der humanitären Sicherheitsexperten verstanden werden als nichtstaatlicher Akteur, der zu strukturellem Wandel im Politikfeld humanitäre Hilfe und dabei zu transnational governance beiträgt (Davis Cross 2013). Erste Überlegungen, eine Gruppe von Sicherheitsexperten im Politikfeld humanitäre Hilfe als epistemische Gemeinschaft zu verstehen, wurden bereits geäußert (Bollettino 2008: 272-273). Doch es existieren bisher keine Untersuchungen darüber, warum Sicherheitsexperten als eine solche epistemische Gemeinschaft verstanden werden können, welche Personen genau zu dieser Gemeinschaft gehören und was diese Gemeinschaft intern zusammenhält, warum diese Gemeinschaft über ausreichend Expertise und vor allem Autorität verfügt, um Wandel herbeizuführen, und wie sie konkret zu Veränderungen im Politikfeld beiträgt. Diese Punkte sind allerdings nicht nur mit Blick auf humanitäre Hilfe unterforscht, sondern ganz allgemein wissen wir wenig über die Entstehung und Funktionsweisen epistemischer Gemeinschaften. Der vorliegende Beitrag trägt dazu bei, diese Fragen anhand der epistemischen Gemeinschaft humanitärer Sicherheitsexperten zu beantworten und reiht sich dabei in die aktuelle Debatte (Davis Cross 2013) über die Weiterentwicklung des Konzeptes der epistemischen Gemeinschaft in den Internationalen Beziehungen (Haas 1992a) ein. Dabei folgt die Untersuchung bisheriger Arbeiten zu epistemischen Gemeinschaften dahingehend, dass über die Analyse einer konkreten epistemischen Gemeinschaft verallgemeinerbare Aussagen für die Rolle und Funktion epistemischer Gemeinschaften im Allgemeinen getroffen werden (siehe zum Beispiel Haas 1992b; Galbreath/McEvoy 2013).

Hierfür wurden zwischen November 2009 und Mai 201227 leitfadengestützte qualitative Interviews geführt. Das Sample besteht aus zwölf Sicherheitsmanagern

6 Synonym finden sich auch die Bezeichnungen Sicherheitsdirektor oder -berater. Hier wird durchgängig der Begriff Sicherheitsmanager verwandt. Aus Gründen der Vereinfachung wird hier einheitlich die männliche Schreibweise verwandt, gleichwohl es auch Sicherheitsdirektorinnen, -managerinnen und -beraterinnen gibt. Auch für andere Personenbezeichnungen wurde aus Gründen der Vereinfachung durchgehend die männliche Form gewählt. 
großer und mittlerer US-amerikanischer und europäischer humanitärer $\mathrm{NGOs}^{7}$ einem Verwaltungsmitarbeiter einer humanitären NGO, sieben Mitarbeitern von NGO-Netzwerken, fünf freiberuflichen oder angestellten Beratern, einem Vertreter einer nationalen Regierung und einem Vertreter einer internationalen Geberorganisation. ${ }^{8}$ Die Interviewpartner wurden einerseits ausgewählt, weil sie innerhalb und außerhalb humanitärer NGOs als Sicherheitsexperten anerkannt werden, teilweise an Standardpublikationen zum Sicherheitsmanagement humanitärer NGOs mitgearbeitet haben und/oder entscheidend an der Institutionalisierung von Kooperationen humanitärer NGOs zu Sicherheitsthemen beteiligt sind und somit zur hier behandelten epistemischen Gemeinschaft gezählt werden können, oder weil sie andererseits einen guten Überblick über die Entwicklungen des Sicherheitsmanagements humanitärer NGOs haben. Somit umfasst das Sample sowohl Mitglieder der hier untersuchten epistemischen Gemeinschaft als auch Nicht-Mitglieder.

Die Interviews entsprechen der Form des »problemzentrierten Interviews« (Witzel 2000; Mayring 2002: 67-72). Sie wurden auf Basis eines Interviewleitfadens geführt, der über die Dauer der Datenerhebung angepasst wurde (Mayring 2002: 67-71; Froschauer/Lüger 2003: 28-34). Da die Interviewpartner über alle Erdteile verteilt arbeiten und leben, wurden die meisten Interviews per Telefon oder über Skype geführt. Sie wurden anonymisiert, transkribiert ${ }^{9}$ und mittels einer qualitativen Inhaltsanalyse ausgewertet (Hermann 2009; Mayring 200: 114-121). Dabei wurden sowohl deduktiv auf Basis der Literatur zum Sicherheitsmanagement humanitärer NGOs (z.B. Van Brabant 2000; 2001a; 2001b; 2010; Bollettino 2008; Stoddard/Harmer 2010) als auch induktiv aus dem Material heraus Kategorien gebildet. Deren Ergebnisse wurden zusammengefasst und für einzelne Befunde repräsentative und besonders prägnante Aussagen ausgewählt. Anschließend an die Interviews erfolgte ein schriftlicher E-Mail-Wechsel mit einigen Interviewpartnern zwecks Präzisierung oder um Informationen über die weitere Entwicklung des Sicherheitsmanagements zu erhalten.

Die Analyse zeigt, dass epistemische Gemeinschaften nicht nur primär aus Wissenschaftlern bestehen, sondern sich aus Personen mit unterschiedlichem beruflichen Hintergrund und/oder aus unterschiedlichen Sektoren (Markt, Staat, Dritter Sektor) zusammensetzen können. Epistemische Gemeinschaften nehmen weiterhin nicht nur Einfluss auf staatliche Akteure, sondern setzen Standards für nichtstaatliche Akteure, die von Letzeren auch anerkannt werden. Dabei wird deutlich, dass

7 Entsprechend einer Publikation des Active Learning Network for Accountability and Performance in Humanitarian Action können humanitäre NGOs entsprechend ihres Budgets in fünf Gruppen eingeteilt werden (Harvey et al. 2010: 20). Die interviewten NGO-Sicherheitsmanager arbeiten für NGOs der Kategorien eins bis vier.

8 Dabei handelt es sich jeweils um die Position, die die Interviewpartner zum Zeitpunkt des Interviews innehatten. Zahlreiche Interviewpartner haben aber in ihrem bisherigen Berufsleben für verschiedene Organisationen gearbeitet.

9 Somit werden die im Folgenden zitierten Aussagen weder einer Person noch einer Organisation zugeordnet. Sie werden einheitlich als Interview mit einem NGO-Sicherheitsexperten und dem jeweiligen Datum angegeben. Zwar wurden auch Sicherheitsexpertinnen interviewt, aber in diesem Aufsatz wird aus Gründen der Anonymität einheitlich die männliche Form verwandt. 
ein Zusammenhang existiert zwischen den Faktoren, die für den internen Zusammenhalt einer epistemischen Gemeinschaft entscheidend sind und der Einflussnahme einer epistemischen Gemeinschaft.

Im Folgenden wird zunächst der Forschungsstand zu epistemischen Gemeinschaften rekapituliert und näher erläutert, wie die vorliegende Analyse zur theoretischen Weiterentwicklung des Konzepts beiträgt. Daran anschließend wird erklärt, warum Sicherheit für humanitäre NGOs ein so schwieriges Thema ist und es daher besonders engagierter Akteure bedarf, um die Professionalisierung des Sicherheitsmanagements und entsprechende Standardsetzung sowohl innerhalb einzelner NGOs als auch organisationsübergreifend voranzutreiben. Schließlich wird erörtert, warum die hier behandelte Gruppe von Sicherheitsexperten als epistemische Gemeinschaft verstanden werden kann, was diese epistemische Gemeinschaft intern zusammenhält, und wie sie zu Wandel beiträgt.

\section{Epistemische Gemeinschaften: Stand der Forschung und aktuelle Debatte zur konzeptionellen Weiterentwicklung}

Entsprechend der Definition von Peter Haas kann eine epistemische Gemeinschaft definiert werden als »a network of professionals with recognized expertise and competence in a particular domain and authoritative claim to policy-relevant knowledge within that domain or issue-area « (Haas 1992a: 3). Im Unterschied zu den meisten älteren Analysen epistemischer Gemeinschaften, bei denen der Fokus hauptsächlich auf Wissenschaftlern und wissenschaftlicher Expertise liegt (Adler 1992; Haas 1992b; Ikenberry 1992; Peterson 1992), wird in der aktuellen Debatte zur Rekonzeptionalisierung, der diese Analyse folgt, davon ausgegangen, dass sich epistemische Gemeinschaften aus Personen mit unterschiedlichem professionellen Hintergrund zusammensetzen können (Davis Cross 2013; s. auch bereits Drake/ Nicolaïdis 1992). Dieses Verständnis epistemischer Gemeinschafen ermöglicht es, Koalitionen von Individuen über Sektoren (Gesellschaft, Staat, Markt) hinweg und damit a priori heterogene Gruppen - als politische Akteure, die ein »common policy enterprise« (Haas 1992a: 3) haben, zu untersuchen. Bislang fanden solch heterogene Zusammenschlüsse meist nur im Konzept transnationaler Advokatennetzwerke (TANs) Berücksichtigung (Keck/Sikkink 1998). Im Gegensatz zu Letzteren setzen sich epistemische Gemeinschaften jedoch nicht aus anderen kollektiven Akteuren zusammen, sondern aus einzelnen Individuen. Diese sind auch keine Advokaten für das Anliegen primär anderer Akteure, sondern in einem hohen Maße selbst von der Sache, um die es geht, betroffen. Sie handeln jedoch nicht (nur) aus Eigennutz, sondern »are ultimately concerned with outward policy outcomes that benefit society [...]« (Davis Cross 2013: 157).

Die Mitglieder einer epistemischen Gemeinschaft verbindet außer ihrer professionellen Expertise, einer persönlichen Betroffenheit und ihrem Engagement auch geteilte Annahmen über Kausalzusammenhänge und geteilte Wertvorstellungen (Haas 1992a: 3). Somit gehen epistemische Gemeinschaften auch über »commu- 
nities of practice« (Wenger et al. 2002) hinaus, da Letztere die Bedeutung der Initiative Einzelner zugunsten einer gewissen durch Praktiken erzeugten Pfadabhängigkeit vernachlässigen (Davis Cross 2013: 147) und weder geteilte Wertvorstellungen noch ein gemeinsames politisches Ziel voraussetzen. Aus der Kombination der genannten Faktoren, die eine epistemische Gemeinschaft auszeichnen, ergibt sich ein spezifischer »shared worldview« (Davis Cross 2013: 147) und ein entsprechendes professionelles Selbstverständnis, die für die Mitglieder der epistemischen Gemeinschaft handlungsleitend sind, die epistemische Gemeinschaft nach innen zusammenhalten und von anderen Akteuren abgrenzen (Davis Cross 2013). Mai'a Davis Cross (2013: 147) zufolge entsteht dieser Zusammenhalt durch eine gemeinsame Sozialisation und geteilte Wissensbestände (s. auch Antoniades 2006: 26). Damit kann einerseits erklärt werden, wie epistemische Gemeinschaften entstehen - ein Aspekt der in der Literatur bisher wenig Berücksichtigung fand, da epistemische Gemeinschaften meist als gegeben angenommen wurden. Andererseits wird durch die Betonung einer gemeinsamen Sozialisation und geteilter Wissensbestände erklärt, was eine Gruppe von Personen aus unterschiedlichen Sektoren und/oder mit unterschiedlichen beruflichen Werdegängen zusammenhält.

Entsprechend richtet die vorliegende Analyse epistemischer Gemeinschaften den Blick auf deren interne Verfasstheit, die sie konstituierenden Faktoren und die darüber erfolgende Abgrenzung von anderen Akteuren, was in der Forschung zu epistemischen Gemeinschaften bisher vernachlässigt wurde. Dabei ist das Konzept der sozialen Identität, dem insbesondere in konstruktivistischen und poststrukturalistischen Ansätzen in den Internationalen Beziehungen eine konstituierende Rolle zugesprochen wird, ein hilfreiches Analyseinstrument. Über soziale Identität wird die Zugehörigkeit von Akteuren zu sozialen Gruppen bestimmt und somit werden ingroups und out-groups geschaffen. Hierzu tragen Identitäten bei, indem sie die »(1) rules of membership that decide who is and is not a member of the category « (Fearon/Laitin 2000: 848) bestimmen und darüber hinaus »(2) sets of characteristics [...] thought to be typical of members of the category, or behaviors expected or obliged of members in certain situations (roles) « (Fearon/Laitin 2000: 848) festlegen.

Haben Arbeiten zu epistemischen Gemeinschaften üblicherweise deren Einfluss auf staatliche Entscheidungsträger untersucht (Adler 1992; Ikenberry 1992), so wird hier gezeigt, dass eine epistemische Gemeinschaft auch und sogar in erster Linie andere nichtstaatliche Akteure beeinflussen kann (Davis Cross 2013). Da immer mehr Aufgaben von privaten Akteuren übernommen werden, wird auch deren Regulierung immer wichtiger. Eine epistemische Gemeinschaft verfügt in diesem Kontext über Autorität, wenn die von ihr formulierten Standards von anderen nichtstaatlichen Akteuren, die nicht an der Formulierung beteiligt waren, akzeptiert werden (Graz/Nölke 2008: 2). Diese Standards können im Sinne einer Selbstregulierung nichtstaatlicher Akteure im Zuge von transnationaler governance verstanden werden, aber auch von Regierungen anerkannt werden und somit zu staatlicher Regulierung beitragen. Daher sind epistemische Gemeinschaften ein Beispiel dafür, wie Wissen zu politischer Einflussnahme führt (Davis Cross 2013: 137). 
Die Standardsetzungsprozesse epistemischer Gemeinschaften unterscheiden sich von anderen Konzeptionalisierungen transnationaler Prozesse der Standardsetzung, zum Beispiel Multistakeholderprozessen oder public-private partnerships (Börzel/ Risse 2005) wie der World Commission on Dams (Dingwerth 2005) oder privateprivate partnerships wie dem Forest Stewardship Council (Arts 2006; Pattberg 2004). In diesen Prozessen haben die vertretenen Akteure zwar alle das gleiche Ziel (Regulierung), aber sie behalten ihre sektorspezifische Identität bei und bilden kein gemeinsames professionelles Selbstverständnis aus. Doch gerade angesichts so genannter revolving door-Effekte zwischen Wirtschaft und Staat, aber auch Drittem Sektor, bei denen Personen im Laufe ihres Berufslebens aufgrund ihrer Expertise in unterschiedlichen Sektoren arbeiten, bilden sich sektorübergreifende Koalitionen aus Personen - deren Zugehörigkeit zu einem Bereich während des Regelsetzungsprozesses wechseln kann - um zum Beispiel in der Wirtschafts- und Finanzpolitik (Seabrooke/Tsingou 2009) zu transnationaler governance beizutragen.

Dabei stellt sich die Frage, wieso es zu einer zwangsfreien Anerkennung der Autorität einer epistemischen Gemeinschaft durch die von ihrer Standardsetzung betroffenen Akteure kommt (Hall/Biersteker 2002: 5; Nölke 2004: 155; Graz/Nölke 2008: 2). Wenn es sich bei Letzteren in erster Linie um nichtstaatliche Akteure handelt, muss die Beziehung zwischen epistemischer Gemeinschaft und Entscheidungsträgern im Unterschied zu bisherigen Analysen, die eine Einflussnahme epistemischer Gemeinschaften auf Regierungsvertreter fokussieren, neu gedacht werden. Hierzu soll die Anerkennung von Autorität als ein sozialer Prozess verstanden werden (Antoniades 2006: 26). Entsprechend wird die Autorität einer epistemischen Gemeinschaft nicht nur als unmittelbare Folge ihrer Expertise aufgefasst, sondern sie ist abhängig von den jeweiligen »knowledge structures«, in welche die epistemische Gemeinschaft eingebettet ist (Andoniades 2006: 26) bzw. dem »worldview« (Davis Cross 2013: 147) der von der Standardsetzung betroffenen Akteure.

Somit wird hier angenommen, dass für die Beantwortung der Frage, warum die epistemische Gemeinschaft humanitärer Sicherheitsexperten über ausreichend Autorität verfügt, um Standards zu setzen, nicht so sehr der Inhalt der Standards von Bedeutung ist, sondern dass vielmehr entscheidend ist, dass diese Standards dem »worldview « (Davis Cross 2013: 147) der von den Standards betroffenen Akteure entsprechen (Antoniades 2006: 26). Die Kombination aus Expertise und Wertvorstellungen begründet also nicht nur den internen Zusammenhalt der epistemischen Gemeinschaft, sondern auch ihre Autorität nach außen (Davis Cross 2013). Daraus folgt, dass genauer bestimmt werden muss, an welche Akteure sich die Standardsetzung der epistemischen Gemeinschaft richtet, und um welche Art von Wissen es sich handelt. Daraus folgt auch, dass die Instrumente, die der epistemischen Gemeinschaft zur Verfügung stehen, um andere nicht-staatliche Akteure von ihrer Standardsetzung zu überzeugen, in den Bereich des arguing fallen. Daher muss der Diskurs der epistemischen Gemeinschaft eine besondere Beachtung erfahren (Fearon/Laitin 2000: 848). 
Entsprechend ihres common policy enterprise strebt die epistemische Gemeinschaft einerseits innerorganisatorische Veränderungen und andererseits einen institutionellen Wandel im Politikfeld humanitärer Hilfe an. Auf Basis der Literatur zu Organisationswandel aus der Perspektive von Professionen (Daudigeos 2013; Ritzer 1975) und Organisationslernen (Ebrahim 2005; Edwards 1997; Argyris/Schön 2007; Van Brabant 2001a) sowie der Literatur zu anderen transnationalen Standardsetzern, wie zum Beispiel Advokatennetzwerken (Keck/Sikkink 1998), können die Strategien der epistemischen Gemeinschaft, um Widerstände der »humanitären Community« für Wandel zu überwinden, identifiziert werden. Die epistemische Gemeinschaft treibt innerhalb der »humanitären Community« zunächst die Institutionalisierung vor allem der Kooperation humanitärer Helfer und humanitärer Organisationen voran. Diese institutionalisierten Zusammenschlüsse können weder als Märkte verstanden werden, weil innerhalb dieser Strukturen Kooperation gegenüber Wettbewerb überwiegt (Seybolt 2009: 1029). Sie sind auch keine Hierarchien, weil sie über keine Zwangsmittel verfügen, um Standards durchzusetzen. Vielmehr können diese institutionalisierten Zusammenschlüsse als Netzwerke verstanden werden (Seybolt 2009), definiert als Koalitionen humanitärer Akteure, die in Sicherheitsfragen kooperieren (Pappi 1993: 84-94; Topçu 1999: 8-9).

Im Rahmen dieser Strukturen kann die epistemische Gemeinschaft dann die unterschiedlichen für die Etablierung und Umsetzung professioneller Standards verantwortlichen Personengruppen ansprechen, um sie von der Relevanz eines professionellen Sicherheitsmanagements für humanitäre NGOs zu überzeugen. Um die hierfür hinderlichen in der »humanitären NGO-Community« vorherrschenden Paradigmen zu überwinden, bedient sich die epistemische Gemeinschaft Strategien der information politics im Sinne der Verbreitung policy-relevanter Informationen, der accountability politics, indem Akteure auf Basis der von ihnen bereits gemachten Erklärungen und Eingeständnisse zur Verantwortung gezogen werden (Keck/ Sikkink 1998: 18-25) und des Framing, womit »conscious strategic efforts by groups of people to fashion shared understandings of the world and of themselves that legitimate and motivate collective action « (McAdam et al. 1996: 6; siehe auch Rein/Schön 1991: 263) gemeint sind. Dadurch gelingt es der epistemischen Gemeinschaft, einen veränderten Umgang mit Sicherheit im Sinne eines "new thinking« auf Basis von Paradigmenwechseln zu etablieren (Faleg 2012: 161).

Zusammenfassend können epistemische Gemeinschaften für die nachfolgende Analyse bestimmt werden als Zusammenschluss aus Individuen aus unterschiedlichen Sektoren mit unterschiedlichem beruflichen Hintergrund. Aus deren gemeinsamer Sozialisation und geteilten Wissensbeständen resultiert ein shared worldview, der sowohl geteilte professionelle Expertise als auch bestimmte Wertvorstellungen und geteilte Annahmen über Kausalzusammenhänge umfasst. Diese Faktoren erklären nicht nur den internen Zusammenhalt einer epistemischen Gemeinschaft, sondern auch ihre Autorität. Damit eine epistemische Gemeinschaft als legitimer Standardsetzer von anderen nichtstaatlichen Akteuren anerkannt wird, muss sie nicht nur intern einen shared worldview haben, sondern auch mit den Zielgruppen ihrer Standardsetzung einen solchen teilen. 
Das auf diese Art neu formulierte Konzept der epistemischen Gemeinschaft könnte daher ein hilfreiches Analyseinstrument im Rahmen der aktuellen auf Foucault zurückgehenden governmentality-Diskussion (z.B. Sending/Neumann 2006; Abrahamsen/Williams 2011: 66) sein, weil es einerseits die Bedeutung von Wissen betont und andererseits keine staatszentrierte Perspektive einnimmt, also nicht auf der strikten Trennung zwischen privaten und öffentlichen Akteuren und Bereichen beruht, sondern die Zugehörigkeit zu einer epistemischen Gemeinschaft an einem bestimmten professionellen Selbstverständnis festmacht.

\section{Humanitäre Identität und Sicherheit}

Humanitäre Identität ist nicht nur auf der individuellen Ebene der Sicherheitsexperten relevant, sondern auch auf Organisationsebene. Die Zusammenhänge zwischen humanitärer Identität und Sicherheit sind vielfältig. Die für die humanitäre Identität konstitutiven Prinzipien bedingen einerseits den Umgang humanitärer NGOs mit Sicherheit. Andererseits ist die humanitäre Identität durch die zunehmende Unsicherheit - aber nicht nur durch diese - in Frage gestellt (Barnett/Weiss 2008b: 5; Vaughn 2009: 264). Dies erfordert zwar eine aktive Auseinandersetzung humanitärer NGOs mit ihrer Sicherheit, erschwert aber eine solche Auseinandersetzung zugleich, da jene die konstituierenden Prinzipien humanitärer Arbeit betrifft. Der epistemischen Gemeinschaft humanitärer Sicherheitsexperten gelingt es, die damit verbundenen Widerstände zu überwinden bzw. den Widerspruch aufzulösen, indem sie argumentiert, dass humanitäre NGOs gerade aufgrund ihrer Identität ein eigenes auf sie zugeschnittenes Sicherheitsmanagement benötigen. Wie der epistemischen Gemeinschaft dies gelingt, wird in den nachfolgenden Kapiteln gezeigt. Hier wird zunächst der Zusammenhang zwischen humanitärer Identität und Sicherheit erläutert.

Soziale Identitäten legen Kriterien für die Zugehörigkeit eines Akteurs zu einer bestimmten Gruppe sowie als typisch erachtete Charakteristika für die Mitglieder dieser Gruppe fest (Fearon/Laitin 2000: 848). Im hiesigen Kontext sind die Mitglieder der Kategorie humanitäre NGO nicht-profitorientierte Nichtregierungsorganisationen, die humanitäre Hilfe durchführen. Darunter fallen die Bereitstellung von Nahrung, Schutzunterkünften, (Trink-)Wasser, Sanitärwesen und medizinischer Hilfe sowie die Betreuung, Versorgung und der Schutz von Flüchtlingen und Vertriebenen (z.B. das Management von Flüchtlingslagern), der Aufbau grundlegender Infrastruktur (z.B. Krankenhäuser), Präventionsmaßnahmen (z.B. Gesundheitsaufklärung, Impfkampagnen oder Katastrophenvorsorge) und die Minenräumung (Bronner 2003: 10; UN 1999). Humanitäre Hilfe grenzt sich dabei idealtypisch von der Entwicklungszusammenarbeit ab, da sie ursprünglich keine strukturbildenden Maßnahmen beinhaltet und nicht auf die Umgestaltung der gesellschaftlichen Verhältnisse abzielt. Doch bereits die Liste humanitärer Aufgaben zeigt, dass die Trennung zwischen humanitärer Hilfe einerseits und Entwicklungszusammenarbeit andererseits nicht immer eindeutig ist. So bieten auch zahlreiche Organisationen 
sowohl humanitäre Hilfe als auch Entwicklungshilfe an, vor allem große internationale NGOs wie CARE, World Vision, Save the Children oder Oxfam. ${ }^{10}$

Trotz der bestehenden Unterschiede zwischen den NGOs basiert die Identität humanitärer NGOs auf den Prinzipien der Menschlichkeit, Neutralität, Unparteilichkeit und Unabhängigkeit (Barnett 2009: 623), die als »set [...] of characteristics« (Fearon/Laitin 2000: 848) im obigen Sinne verstanden werden können. Entsprechend dem ersten Grundsatz sind humanitäre Helfer bestrebt, "menschliches Leiden überall und jederzeit zu verhüten und zu lindern [sowie] Leben und Gesundheit zu schützen und der Würde des Menschen Achtung zu verschaffen« (DRK 2013). Dabei handeln sie zumindest teilweise aus uneigennützigem Antrieb (Fearon 2008: 51). Neutralität bedeutet, dass sich humanitäre NGOs der »Teilnahme an Feindseligkeiten wie auch, zu jeder Zeit, an politischen, rassischen, religiösen oder ideologischen Auseinandersetzungen« (DRK 2013) enthalten. Des Weiteren soll humanitäre Hilfe entsprechend der Grundsätze der Unparteilichkeit und der Unabhängigkeit ungeachtet von »Nationalität, Rasse, Religion, sozialer Stellung oder politischer Überzeugung« (DRK 2013) und unabhängig von Regierungen gewährt werden (DRK 2013).

Die humanitären Prinzipien bedingen nicht nur die Identität humanitärer NGOs, sondern auch deren Umgang mit Sicherheit. NGOs gehen traditionell davon aus, dass eine an diesen Prinzipien ausgerichtete Hilfe automatisch dazu führt, dass sie sowohl von den Hilfsbedürftigen als auch allen Konfliktparteien akzeptiert werden und dass dies ihre Sicherheit gewährleistet (Fast/O'Neill 2010: 5). Dieser Ansatz wird als Akzeptanz-Ansatz bezeichnet (Martin 1999: 5; Van Brabant 2000: 57-67), und er wurde traditionell als ein passiver Ansatz verstanden, der keinen aktiven Beitrag einer NGO, der über ein an humanitären Prinzipien ausgerichtetes Handeln hinausgeht, erfordert (Fast/O’Neill 2010: 5; Fast et al. 2013: 223-227). Da humanitäre Helfer aber, besonders in einigen Regionen, immer häufiger und teilweise gezielt Opfer gewaltsamer Übergriffe werden (Stoddard et al. 2006: 1; 2009: 1-11; 2011: 1-11), wird deutlich, dass die humanitären Prinzipien keinen automatischen Schutz mehr darstellen (Vaughn 2009: 263). Darauf aufbauend formulieren humanitäre NGOs im Sinne einer »Versicherheitlichung« eine existentielle Bedrohung für ihren besonderen Typ von Organisation, die unmittelbare Maßnahmen zur Abhilfe rechtfertigt (Vaughn 2009). Dies soll ihnen besondere Aufmerksamkeit sichern.

Gleichzeitig überdeckt dieser Diskurs, der die Ursachen für die Unsicherheit auf externe Faktoren zurückführt, die Notwendigkeit für humanitäre NGOs, sich intensiv mit ihrem eigenen Verhalten und Umgang mit Sicherheit auseinanderzusetzen. Denn einige Beobachter, auch aus der »NGO-Community«, argumentieren, dass die Politisierung und Militarisierung humanitärer Hilfe (Donini 1996: 191), wobei Hilfe im Rahmen von Strategien im Sinne von winning hearts and minds in militä-

10 Des Weiteren haben sich in den letzten Jahren auch solche Verständnisse humanitärer Hilfe entwickelt, die sehr wohl auf die Beseitigung von Konfliktursachen abzielen (Barnett/Snyder 2008: 150-154). 
rische Konzepte integriert wird, um die Akzeptanz des militärischen Einsatzes bei der lokalen Bevölkerung zu erhöhen (Macrae 2002: 9-10), zu einer Verwischung der Grenzen zwischen militärischen Akteuren und Handlungen auf der einen Seite und zivilen, humanitären und entwicklungsorientierten Akteuren und Handlungen auf der anderen Seite (Smillie/Minear 2004: 88) führe, wodurch zivile Helfer im schlimmsten Falle zu Angriffszielen werden (Lieser 2007, 53-54; Radtke 2008: 2; Runge 2009: 20-23; Torrente 2004). Auch wenn solche Kausalzusammenhänge zwischen der Militarisierung und Politisierung von Hilfe und der zunehmenden Gewalt gegen Hilfsorganisationen, die Larissa Fast (2010) deep-causes-Erklärungen nennt, kritisch hinterfragt werden, da sie nur sehr schwer empirisch nachweisbar sind (Fast 2010: 3), deuten sie doch auf einen Zusammenhang zwischen der Aufgabe der Unparteilichkeit und Unabhängigkeit durch die Zusammenarbeit mit Konfliktparteien und Sicherheit hin. Allerdings können sie auch dazu führen, dass die Verletzung humanitärer Prinzipien als alleinige Ursache für Unsicherheit gesehen wird, und dass NGOs sich auf das passive Befolgen der humanitären Prinzipien als Sicherheitsstrategie beschränken, davon ausgehend »that doing good programming will win the consent of the local population and acceptance will automatically follow« (Fast/O’Neill 2010: 5). So ist es für NGOs schwierig zu akzeptieren, dass sie trotz ihrer guten Absichten Opfer von Gewalt werden. Ein Interviewpartner erklärte zum Beispiel: »[It] is difficult in this business to point fingers and attribute fault because we're trying to do good, therefore how can you criticize me? «11 Doch NGOs werden auch ohne dass sie ihre Unabhängigkeit oder Unparteilichkeit aufgeben zu Opfern von Gewalt, so dass humanitäre NGOs ein weitaus umfassenderes und vor allem aktives Sicherheitskonzept benötigen (Fast/O'Neill 2010: 5). Somit wird die humanitäre Identität durch die Unsicherheit einerseits bedroht (Vaughn 2009: 264) $)^{12}$ und andererseits behindert diese Identität eine Auseinandersetzung der NGOs mit der Unsicherheit, da NGOs hierfür die ihre Arbeit und ihr Selbstverständnis konstituierenden Prinzipien hinterfragen müssen.

Dabei befürchten NGOs entsprechend ihrem Fokus auf der Linderung akuter Not (Van Brabant 2001a: 189) auch, dass aktive Sicherheitsmaßnahmen ihre eigentliche humanitäre Arbeit, also die Durchführung humanitärer Projekte, behindern könnten. ${ }^{13}$ Entsprechend existieren organisationsinterne Hindernisse für eine aktive Auseinandersetzung mit Sicherheit, sowohl bei einzelnen humanitären Helfern als auch auf der Ebene des Managements humanitärer NGOs. Dies betrifft unter anderem die Weitergabe von Informationen über Sicherheitsvorfälle, zum Beispiel von den Einsatzgebieten zu den Hauptquartieren. Entsprechende Hindernisse basieren unter anderem auf grundsätzlichen Spannungen zwischen der Ebene der Einsatzgebiete und der Ebene der Hauptquartiere, die aus unterschiedlichen Interessen und Ansichten resultieren (Suzuki 1998: 4-6). Die Mitarbeiter humanitärer NGOs fürchten, dass ihr Hauptquartier die Durchführung eines Projektes beendet, wenn es von

11 Interview mit einem NGO-Sicherheitsexperten, 31. August 2011.

12 Interview mit einem NGO-Sicherheitsexperten, 31. August 2011.

13 Van Brabant (2001b: 34), siehe auch Interview mit einem NGO-Sicherheitsexperten, 09. September 2011. 
einem Sicherheitsvorfall erfährt (Van Brabant 2001b: 34). Daher werden Sicherheitsvorfälle oftmals weder innerhalb der NGOs gemeldet noch werden entsprechende Informationen unter den NGOs geteilt. Dies hat auch damit zu tun, dass Sicherheitsvorfälle oftmals auf individuelles Fehlverhalten humanitärer Helfer zurückgeführt werden, und nicht auf strukturelle Gründe: »This reflects an organisational culture which sees >critical review $<$ and >evaluation< as fault-finding and punitive, rather than as learning opportunities« (Van Brabant 2001b: 34). Entsprechend erklärt ein Interviewpartner: »There are many organisations on the ground that don't have security professionals and are frankly, I think, in denial about what their needs are «. ${ }^{14}$ Nach Ansicht eines Experten existiert in den Führungsetagen humanitärer NGOs eine grundsätzliche Abneigung gegenüber Sicherheitsmanagement und Sicherheitsmanagern (Renouf 2011: 156):

»General management doesn't really want to see this. They see security as an add-on, something that is nice to have [...] but the view has been that it is something that impedes operations, that impedes access to beneficiaries, that impedes programme implementation, therefore it impedes spending the money that donors have been giving us. And the security managers have a hard time combating that, because [security] has always been seen as an end in itself. ${ }^{15}$

Aber auch auf Ebene einzelner Mitarbeiter gibt es Widerstände gegen die Etablierung eines Sicherheitsmanagements. So würden manche schlicht ihren Kopf in den Sand stecken und hoffen, dass sie von Angriffen oder anderen Sicherheitsvorfällen verschont bleiben. ${ }^{16}$

Trotz dieser organisationsinternen mit der humanitären Identität zusammenhängenden Hindernisse für die Auseinandersetzung mit ihrer Sicherheit haben viele NGOs heutzutage im Vergleich zu vor zehn bis fünfzehn Jahren eine Sicherheitspolitik (Van Brabant 2010: 5). Diese sollte folgende Elemente umfassen: »A general statement acknowledging risk in aid work; a clarification of what is meant by $[\ldots]$ security; [...] basic principles in the organisation's philosophy and practice with regard to security management [...] (Van Brabant 2001b: 28). Daraus ergeben sich idealerweise konkrete Verfahren für die Durchführung von zum Beispiel einer Analyse der Sicherheitslage oder einer Evakuierung (zum Beispiel Macpherson 2004: 9-46), die handlungsleitend für die Mitarbeiter der NGO sein sollen. Entsprechende Verfahren und Abläufe können auch in Sicherheitshandbüchern niedergelegt sein (zum Beispiel ICRC 2007; Macpherson 2004). Des Weiteren haben zahlreiche NGOs interne Strukturen zum Sicherheitsmanagement etabliert und bieten zum Beispiel Sicherheitstrainings für ihre Mitarbeiter an oder haben Sicherheitsmanager eingestellt (Van Brabant 2010: 5; Bickley 2006: 4; Mujawar 2009). In vielen NGOs hapert es jedoch an einem Mainstreaming von Sicherheit, so dass die einzelnen Politiken, Prozesse, Strukturen und Praktiken nicht unbedingt in ein kohärentes System integriert sind (INSSA 2013c). ${ }^{17}$ Insgesamt kann auf Basis der Interviews

14 Interview mit einem NGO-Sicherheitsexperten, 23. August 2011.

15 Interview mit einem NGO-Sicherheitsexperten, 31. August 2011.

16 Interview mit einem NGO-Sicherheitsexperten, 23. August 2011.

17 Interview mit einem NGO-Sicherheitsexperten, 12. April 2012. 
und der Literatur festgehalten werden, dass große Unterschiede zwischen den NGOs existieren mit Blick auf Sicherheitspolitiken, -verfahren und -strukturen (Mujawar 2009; Fast et al. 2013: 235, Humanitarian Practice Network 2010a: 1). Doch es gibt auch immer noch zahlreiche NGOs, die über gar kein oder nur über ein rudimentäres Sicherheitsmanagement verfügen (InterAction 2013; Gaul et al. 2006: 32$){ }^{18}$

Die identitätsbasierten Hindernisse für die Auseinandersetzung mit Sicherheit erschweren auch die Kooperation von NGOs in Sicherheitsfragen (Bollettino 2008: 265-266), vor allem hinsichtlich des Informationsaustauschs. Darüber hinaus wird argumentiert, dass strukturelle Faktoren wie der Wettbewerb um Spendengelder die Kooperation zwischen humanitären NGOs begrenzen (Bollettino 2008: 268; Cooley/Ron 2002: 25-31). A priori sollten humanitäre Akteure untereinander unbeschränkt Informationen austauschen, doch in der Realität ist dies nicht immer der Fall: »In the past, organizations have been wary about sharing information with others partly for fear that it compromises their own security contacts, but also because of the general tendency to protect information for their own « (Stoddard/ Harmer 2010: 14). Des Weiteren hat die unzureichende Weitergabe von Informationen auch mit dem mangelnden Vertrauen innerhalb der »humanitären Community« zu tun. So kommt ein Experte zu folgendem Ergebnis: »Anybody who spends some time in the Western-dominated aid world, cannot but be astonished by the pervasive levels of distrust: distrust between people within agencies, between agencies« (Van Brabant 2010: 5).

Entsprechend scheiterten Ende der 1990er Jahre noch mindestens zwei Initiativen zur Etablierung von institutionalisierten Sicherheitskooperationen von NGOs: das International Committee to Protect Aid Workers (White 1997: 17) und das Project for a humanitarian security network (Gallien 1998: 17). Nach Ansicht eines Interviewpartners waren die $»$ NGOs $[. .$.$] not ready at the time { }^{19}{ }^{9}$ und es fehlte ihnen an Einsicht, dass Kooperation letztlich für alle Beteiligten von Nutzen ist. ${ }^{20}$ Ab Mitte der 2000er Jahre wurden jedoch vermehrt institutionalisierte Kooperationen in Sicherheitsfragen etabliert, in Form von Sicherheitsnetzwerken, die den Informationsaustausch ermöglichen und Ressourcen bündeln, um zum Beispiel gemeinsame Sicherheitsmanager einzustellen (Bickley 2006: 1, 5-16). Deren Zunahme sowie die Professionalisierung des Sicherheitsmanagements innerhalb der NGOs trotz zahlreicher Widerstände kann mit den Aktivitäten der epistemischen Gemeinschaft humanitärer Sicherheitsexperten erklärt werden. Dabei können Aussagen über das Entstehen und die Funktionsweise epistemischer Gemeinschaften auch über den untersuchten Fall hinaus gemacht werden. Im Folgenden wird erläutert, warum humanitäre Sicherheitsexperten als epistemische Gemeinschaft verstanden werden können und was diese Gemeinschaft intern zusammenhält.

18 Interview mit einem NGO-Sicherheitsexperten, 26. Januar 2012.

19 Interview mit einem NGO-Sicherheitsexperten, 25. April 2012.

20 Interview mit einem NGO-Sicherheitsexperten, 25. April 2012. 


\section{Die epistemische Gemeinschaft der NGO-Sicherheitsexperten}

Entsprechend der Definition von Peter Haas sind die Mitglieder der epistemischen Gemeinschaft humanitärer Sicherheitsexperten Personen, die sich professionell mit Sicherheitsfragen für NGOs und aus NGO-Perspektive befassen. Sie werden innerhalb der »humanitären NGO-Community« als Experten auf dem Gebiet der NGOSicherheit betrachtet, denn sie sind nach eigener Auskunft und nach Auskunft von Mitarbeitern ihrer jeweiligen NGO sowie anderer NGOs diejenigen, die um Rat gefragt werden, wenn es um Fragen des Sicherheitsmanagements geht. ${ }^{21}$ Darüber hinaus werden die Mitglieder der epistemischen Gemeinschaft auch außerhalb der »NGO-Community« als Sicherheitsexperten angesehen und sind oft Ansprechpartner von Regierungen und den Vereinten Nationen, wenn es um Themen zur NGOSicherheit geht (z.B. Irish Aid 2013: 1; Saving Lives Together 2011a; 2011b). ${ }^{22}$

Das »common policy enterprise« (Haas 1992a: 3) der humanitären Sicherheitsexperten ist die Professionalisierung des Sicherheitsmanagements humanitärer Organisationen, vor allem humanitärer NGOs, sowohl innerhalb der einzelnen Organisationen als auch organisationsübergreifend und das Setzen entsprechender Standards innerhalb der »humanitären Community «. ${ }^{23}$ Somit sind die Mitglieder der epistemischen Gemeinschaft sowohl Experten als auch Entrepreneure. Sie sind bestrebt, die »humanitäre Community« davon zu überzeugen, dass »to have a security culture and to have security responsibles and a proper security policy is essential«. ${ }^{24}$ Ähnlich wie im Falle von Professionen (Daudigeos 2013) kommt dabei der Impuls zur Weiterentwicklung des Sicherheitsmanagements nicht von außen, sondern aus der »humanitären Community« selbst. Die Mitglieder der epistemischen Gemeinschaft können auf Basis ihres professionellen Know-hows Vorschläge für die Etablierung eines Sicherheitsmanagements innerhalb einzelner Organisationen unterbreiten, doch ihnen fehlt die letztliche Entscheidungskompetenz (Daudigeos 2013: 723-725), da ihre Vorschläge von den NGO-Vorständen genehmigt werden müssen, die jedoch oftmals Vorbehalte haben. ${ }^{25}$

Dabei sind die Mitglieder der epistemischen Gemeinschaft selbst von der Etablierung eines professionellen Sicherheitsmanagements betroffen. Sie sind einerseits an der Verbesserung der Situation ihrer Kollegen, die in gefährlichen Einsatzgebieten

21 Interview mit einem NGO-Sicherheitsexperten, 27. November 2009; Interview mit einem NGO-Sicherheitsexperten, 23. August 2011; Interview mit einem NGO-Sicherheitsexperten, 26. Januar 2012.

22 Einige Mitglieder der epistemischen Gemeinschaft wurden von Security Magazine zu den »Most Influential People in NGO Security« 2011 und 2012 gewählt (Security Magazine 2011; 2012).

23 Interview mit einem NGO-Sicherheitsexperten, 23.08.2011; Interview mit einem NGOSicherheitsexperten, 31.08.2011; Interview mit einem NGO-Sicherheitsexperten, 20.10.2011; Interview mit einem NGO-Sicherheitsexperten, 26.01.2012; INSSA 2013a; InterAction 2006.

24 Interview mit einem NGO-Sicherheitsexperten, 15. Dezember 2011; siehe auch Interview mit einem NGO-Sicherheitsexperten, 12. Oktober 2011; Interview mit einem NGO-Sicherheitsexperten, 25. April 2012.

25 Interview mit einem NGO-Sicherheitsexperten, 31. August 2011; Renouf 2011: 156. 
arbeiten, interessiert. Dabei sind sie motiviert durch »passion or belief «, ${ }^{26}$ ihren Wunsch »to be part of creating something that was worth creating $«{ }^{27}$ und ihren Anspruch, dabei für ihre »Community« auf transnationaler Ebene nützlich zu sein ${ }^{28}$ (Davis Cross 2013: 157). Entsprechend findet das Engagement der Mitglieder der epistemischen Gemeinschaft für die Professionalisierung des Sicherheitsmanage-

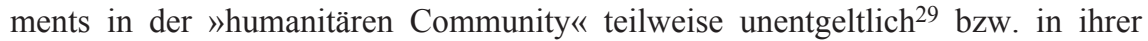
Freizeit $^{30}$ statt. Andererseits handeln die Mitglieder der epistemischen Gemeinschaft nicht nur uneigennützig. Insbesondere angesichts der Widerstände aus den Führungsetagen der NGOs gibt es für sie auch strategische Gründe, die Etablierung von Strukturen des Sicherheitsmanagements voranzutreiben, um ihre Tätigkeit zu legitimieren und entsprechende Arbeitsplätze zu verstetigen. Denn entsprechend der Aussage eines Interviewpartners nimmt die Bereitschaft innerhalb einer NGO, sich mit Sicherheit auseinanderzusetzen, ab, wenn diese Organisation keine Sicherheitsvorfälle zu vermelden hat. ${ }^{31}$

Im Unterschied zum lange Zeit vorherrschenden Verständnis setzt sich die hier behandelte epistemische Gemeinschaft weder ausschließlich noch hauptsächlich aus Wissenschaftlern zusammen, sondern umfasst vor allem Sicherheitsmanager humanitärer NGOs sowie einige Forscher, freiberufliche Berater, Mitarbeiter von privaten und intergouvernementalen Trainings- und Beratungseinrichtungen für humanitäre NGOs sowie Mitarbeiter von Geberinstitutionen. Die Mitglieder der epistemischen Gemeinschaft haben neben ihren derzeit unterschiedlichen Arbeitgebern auch unterschiedliche berufliche Hintergründe. Während einige seit langer Zeit für humanitäre NGOs arbeiten, sind andere erst seit ein paar Jahren im humanitären Sektor aktiv und haben vorher für staatliche oder privatwirtschaftliche Akteure gearbeitet. Dabei stellt sich die Frage, wie Personen mit so unterschiedlichen beruflichen Hintergründen und aus so unterschiedlichen Sektoren eine epistemische Gemeinschaft bilden können bzw. wie angesichts dieser Heterogenität festgelegt werden kann, wer zur epistemischen Gemeinschaft gehört und wer nicht.

Die Zugehörigkeit zur epistemischen Gemeinschaft wird nicht nur durch den Beruf oder die Tätigkeit an sich bestimmt, sondern auch durch »[a] shared set of normative and principled beliefs« sowie »shared causal beliefs« (Haas 1992a: 3). Im hiesigen Falle reicht es also nicht aus, dass die Sicherheitsexperten über technisches Wissen verfügen, zum Beispiel darüber, wie man ein Sicherheitshandbuch oder Evakuierungspläne schreibt, denn dies würde alle Arten von Sicherheitsmanagern einschließen, egal ob sie für eine humanitäre NGO oder zum Beispiel für eine Ölfirma arbeiten. Die Sicherheitsexperten, die zur hier behandelten epistemischen Ge-

26 Interview mit einem NGO-Sicherheitsexperten, 26. Januar 2012; siehe auch Interview mit einem NGO-Sicherheitsexperten, 20. Oktober 2011.

27 Interview mit einem NGO-Sicherheitsexperten, 26. Januar 2012.

28 Interview mit einem NGO-Sicherheitsexperten, 26. Januar 2012.

29 Interview mit einem NGO-Sicherheitsexperten, 31. August 2011.

30 Interview mit einem NGO-Sicherheitsexperten, 12. Oktober 2011; Interview mit einem NGO-Sicherheitsexperten, 20. Oktober 2011; Interview mit einem NGO-Sicherheitsexperten, 26. Januar 2012.

31 Interview mit einem NGO-Sicherheitsexperten, 14. Januar 2010. 
meinschaft zählen, teilen zusätzlich zu ihrer fachlichen Expertise einen gemeinsamen Wertekanon, ${ }^{32}$ woraus sich »a shared worldview« (Davis Cross 2013: 147) darüber ergibt, wie Sicherheitsmanagement speziell humanitärer NGOs ausgestaltet werden sollte. Sie teilen daher ein spezifisches professionelles Selbstverständnis als humanitäre Sicherheitsexperten, das auch als gemeinsame Identität bezeichnet werden kann.

Dies bedeutet, dass sich die Sicherheitsexperten der epistemischen Gemeinschaft im Sinne eines »set of characteristics« (Fearon/Laitin 2000: 848) einerseits den humanitären Prinzipen der Humanität, Neutralität, Unabhängigkeit und Unparteilichkeit verpflichtet fühlen. Andererseits haben sie alle das NGO $»$ mindset $\aleph^{33}$ verinnerlicht und verfügen über »the proper understanding of the aid community«. ${ }^{34}$ Die Bedürfnisse der humanitären NGOs unterscheiden sich nämlich von denen anderer Akteure, die Beratung in Sicherheitsfragen benötigen. Vor allem aufgrund ihrer Identität als neutrale, unabhängige und unparteiliche Akteure sind humanitäre NGOs »a very particular kind of beneficiaries with their own way to deal with security [...] ${ }^{35}$ So fordert zum Beispiel der Verhaltenskodex der International Safety and Security Association (INSSA), einer auf individueller Mitgliedschaft beruhenden Organisation zur Förderung der Professionalisierung des Sicherheitsmanagements innerhalb humanitärer Organisationen, von den Mitgliedern, dass sie »shall strive to the extent possible, to make security management strategies consistent with humanitarian principles and values« (INSSA 2012c). Damit zusammenhängend teilen die Mitglieder der epistemischen Gemeinschaft auch »shared causal beliefs, which are derived from their analysis of practices leading or contributing to a central set of problems in their domain and which then serve as the basis for elucidating the multiple linkages between possible policy actions and desired outcomes« (Haas 1992a: 3). Die Mitglieder der epistemischen Gemeinschaft teilen zum Beispiel die Grundannahme, dass ein passiver Sicherheitsansatz, der auf dem automatischen Schutz als Folge des neutralen, unabhängigen und unparteilichen Status humanitärer NGOs beruht, nicht mehr ausreicht (Van Brabant 2010: 14). Dies basiert auch auf der geteilten Annahme, dass zwischen NGOs wechselseitige Abhängigkeiten (Interdependenzen) hinsichtlich ihrer Sicherheit existieren. ${ }^{36}$ So erklärt ein Handbuch für humanitäre NGOs: »If a number of agencies operate in a district and the majority of them decide to adopt armed guards, this will increase the vulnerability of the remainder, who have now become a comparatively >soft « target« (Humanitarian Practice Network 2010b: 17). Und ein Interviewpartner erläutert:

$»[\mathrm{NGO} A]$ is very different from [NGO B]. But for the other stakeholders, government, media, general public, civil society, more or less, they are considered as one. [...] The action or inaction of one organisation does impact the action or inaction of the other and

32 Interview mit einem NGO-Sicherheitsexperten, 23. August 2011.

33 Interview mit einem NGO-Sicherheitsexperten, 25. April 2012.

34 Interview mit einem NGO-Sicherheitsexperten, 25. April 2012.

35 Interview mit einem NGO-Sicherheitsexperten, 17. November 2011.

36 Humanitarian Practice Network (2010b: 17); Interview mit einem NGO-Sicherheitsexperten, 10. Oktober 2011; Interview mit einem NGO-Sicherheitsexperten, 04. November 2011. 
also the risk profile of the whole sector. There have been some incidents when one INGO was intimidated or attacked [because it was] confused with another NGO. And the work profile of both the INGOs was totally different but, so, this is how they are viewed, as a collective, collective entity. (37 $^{37}$

Sicherheitsexperten, die das NGO mindset nicht verinnerlicht haben und anwenden, werden von den Mitgliedern der epistemischen Gemeinschaft nicht in diese aufgenommen. Bei diesen out-groups handelt es sich vor allem um Sicherheitsmanager mit einem aktuellen oder vormaligen beruflichen Hintergrund im Militär, der Polizei, Geheimdiensten oder Strafverfolgungsbehörden. ${ }^{38}$ Diesen wird von den Mitgliedern der epistemischen Gemeinschaft unterstellt, dass ihnen - a priori - bestimmte »Kernkompetenzen « ${ }^{39}$ fehlen:

»So neither of them orchestrate or manage or build solutions within a resource deprived situation. They carry out, you know, standard physical security, and communication security and many other things but they usually specialise in one area and they are not, you know, ultimately the advisor responsible for the complexities that come with development and humanitarian work. « ${ }^{40}$

Sicherheit für humanitäre NGOs sei hingegen »not [about] guns and high walls [but] much about social engineering sometimes «. ${ }^{41}$ Sicherheitsmanagern mit einer beruflichen Vergangenheit in staatlichen Sicherheitsinstitutionen wie dem Militär oder der Polizei wurde jedoch nicht grundsätzlich die Fähigkeit abgesprochen, sich das NGO mindset anzueignen. ${ }^{42}$ Gelingt ihnen dies, können sie in die epistemische Gemeinschaft aufgenommen und somit Teil der in-group werden. Gelingt ihnen dies jedoch nicht, werden sie auch von der »humanitären NGO-Community « nicht anerkannt. Die Zusammensetzung der epistemischen Gemeinschaft ist somit nicht statisch. Die Mitglieder der epistemischen Gemeinschaft bestimmen selbst, wen sie ihn ihre Gruppe aufnehmen und wen nicht. Dabei ist die Zugehörigkeit nicht formalisiert, und es gibt auch kein Mitgliederregister oder ähnliches, sondern die Mitgliedschaft einer Person in der epistemischen Gemeinschaft weist sich darüber aus, dass die anderen Mitglieder mit dieser Person ihre Informationen teilen und sie an einem entsprechenden Austausch (fernmündlich, schriftlich oder im Rahmen von gemeinsamen Treffen) beteiligen.

Davis Cross (2013: 147) zufolge entsteht der Zusammenhalt einer epistemischen Gemeinschaft und ihr »shared worldview« auf Basis einer gemeinsamen Sozialisation und geteilter Wissensbestände (s. auch Antoniades 2006: 26). Dies trifft auch auf die hier behandelte epistemische Gemeinschaft humanitärer Sicherheitsexperten zu, die sich Ende der 1990er Jahre im Rahmen zweier NGO-Sicherheitstrainings (eines in den USA und eines in Großbritannien), die unter der Ägide des US-ameri-

37 Interview mit einem NGO-Sicherheitsexperten, 10. Oktober 2011.

38 Interview mit einem NGO-Sicherheitsexperten, 25. April 2012.

39 Interview mit einem NGO-Sicherheitsexperten, 26. Januar 2012.

40 Interview mit einem NGO-Sicherheitsexperten, 26. Januar 2012.

41 Interview mit einem NGO-Sicherheitsexperten, 17. November 2011.

42 Interview mit einem NGO-Sicherheitsexperten, 26. Januar 2012; siehe auch Interview mit einem NGO-Sicherheitsexperten, 17. November 2011. 
kanischen NGO-Verbandes InterAction stattfanden, formierte. ${ }^{43}$ Dabei wurde einerseits der Grundstein für einen sich immer weiter entwickelnden bestimmten Wissenskanon zum professionellen Umgang mit dem Sicherheitsmanagement humanitärer NGOs gelegt. Das Wissen der Mitglieder der epistemischen Gemeinschaft wurde somit zumindest teilweise in Form eines "rrational and theoretical training" (Ritzer 1975: 631, bezugnehmend auf Max Weber 2005: 335-336) vermittelt, was den professionellen Charakter der Gruppe ausmacht. Andererseits setzte ein Sozialisationsprozess ein:

»The network that was created through those trainings exists today and there was a consensus there that in the future we would always share our information openly and [...] cite it with attribution appropriately. And that's maintained pretty well so far. $\aleph^{44}$

Angesichts der oben angesprochenen Hindernisse für die Weitergabe von sicherheitsrelevanten Informationen innerhalb der »humanitären Community« stellt sich die Frage, worauf dieser Konsens beruht bzw. wodurch die Informationsweitergabe ermöglicht wird.

Das Beispiel der epistemischen Gemeinschaft humanitärer Sicherheitsexperten zeigt, dass im Rahmen einer gemeinsamen Sozialisation ein Faktor für den Zusammenhalt der epistemischen Gemeinschaft entscheidend ist: Vertrauen. Zwischen den Mitgliedern der epistemischen Gemeinschaft hat sich ein Vertrauen entwickelt, dass sich die anderen an die gemeinsamen Regeln halten. Dieses Vertrauen ist essentiell für den Zusammenhalt der epistemischen Gemeinschaft:

»... the question is basically not so much how can you share it, [...] I mean it is possible to share everything from a technical point of view. But [...] they have to trust each other to do that. And trust is something about persons, individual persons trusting another individual person. $\aleph^{45}$

Das Vertrauen basiert zum einen auf den geteilten Wertvorstellungen der Mitglieder der epistemischen Gemeinschaft: »Well, you build this trust by having some kind of common value set. And that tends to be humanitarian values: neutrality, impartiality and independence $\ll .{ }^{46} \mathrm{Zum}$ anderen entsteht Vertrauen auf Grundlage positiver Erfahrungen aus vorherigen Kooperationen. Dies gilt nicht nur für die Beziehungen zwischen Staaten (Keohane 1984), sondern Margaret Levi und Gillian H. Murphy argumentieren mit Blick auf die Schaffung von Koalitionen aus Aktivisten: $»[\ldots]$ trust beliefs derive from personal relations and networks that provide knowledge of past actions, commitments and reputational investments. Over time strangers involved in a coalition may come to know each other well enough to form trust relations« (Levi/Murphy 2006: 657). Entsprechend bedarf es für eine Vertrauensbildung einer gewissen Dauer der Beziehung. Des Weiteren lässt sich Vertrauen besser in kleinen und konstanten Gruppen aufbauen als in großen Gruppen mit ständig wechselnder Zusammensetzung. Peter Haas’ Definition einer epistemischen

43 Interview mit einem NGO-Sicherheitsexperten, 22. August 2011; siehe auch Interview mit einem Sicherheitsexperten, 01. Dezember 2009.

44 Interview mit einem NGO-Sicherheitsexperten, 22. August 2011.

45 Interview mit einem NGO-Sicherheitsexperten, 12. Oktober 2011.

46 Interview mit einem NGO-Sicherheitsexperten, 22. August 2011. 
Gemeinschaft zufolge handelt es sich bei dieser um eine »concrete collection of individuals« (Haas 1992a: 27) mit einer »relativ kleinen« (Haas 1992a: 27) Anzahl an Mitgliedern. Die epistemische Gemeinschaft der Sicherheitsexperten ist entsprechend auch »a pretty small community [...], so everybody kind of knows everybody $\ll{ }^{47}$ Des Weiteren bedarf es neben Zeit ${ }^{48}$ auch häufiger Interaktion zwischen den betroffenen Personen ${ }^{49}$ und vor allem Strukturen, die persönliche Treffen ermöglichen. Ein Interviewpartner erklärte mit Blick auf die epistemische Gemeinschaft:

$»[\ldots]$ over the years around the world it's the same faces that come up time and time again, you meet the same people in a different country all the time doing the same work, and so you know there are a bunch of people you simply got to know over the years and you get on and you trust each other «. ${ }^{50}$

Einige Interviewpartner bezeichneten ihre Kollegen gar als »Freunde «. ${ }^{51}$ Diese persönlichen Beziehungen ermöglichen eine Kooperation trotz der Tatsache, dass die Mitglieder der epistemischen Gemeinschaft zwischen einzelnen Organisationen oder gar Sektoren wechseln können. ${ }^{52}$

Im Folgenden wird aufgezeigt, wie es der epistemischen Gemeinschaft gelingt, die Professionalisierung und Standardisierung des Sicherheitsmanagements humanitärer NGOs voranzutreiben und warum sie dabei von der »humanitären NGO Community« als legitime Autorität wahrgenommen wird. Dabei wird deutlich, dass die für den internen Zusammenhalt der epistemischen Gemeinschaft entscheidenden Faktoren auch für deren Autorität relevant sind. Die Mitglieder der epistemische Gemeinschaft müssen nicht nur miteinander einen bestimmten worldview teilen, sondern dieser muss auch mit dem worldview derjenigen, an die die Standards gerichtet sind, übereinstimmen.

\section{Professionalisierung und Standardisierung des Sicherheitsmanagements durch Institutionalisierung}

Die epistemische Gemeinschaft humanitärer Sicherheitsexperten hat unterschiedliche Formen der Institutionalisierung von Kooperationen im Politikfeld humanitäre Hilfe im Sinne von Netzwerken und somit den strukturellen Wandel auf interorga-

47 Interview mit einem NGO-Sicherheitsexperten, 26. Januar 2012.

48 Interview mit einem NGO-Sicherheitsexperten, 09. September 2011.

49 Interview mit einem NGO-Sicherheitsexperten, 23. August 2011; Interview mit einem NGO-Sicherheitsexperten, 09. September 2011; Interview mit einem NGO-Sicherheitsexperten, 13. Januar 2012; Interview mit einem NGO-Sicherheitsexperten, 07. Dezember 2011.

50 Interview mit einem NGO-Sicherheitsexperten, 12. April 2012; siehe auch Interview mit einem NGO-Sicherheitsexperten, 26. Januar 2012.

51 Interview mit einem NGO-Sicherheitsexperten, 31. August 2011; Interview mit einem NGO-Sicherheitsexperten, 12. Oktober 2011; Interview mit einem NGO-Sicherheitsexperten, 18. November 2011; Interview mit einem NGO-Sicherheitsexperten, 12. April 2012.

52 Interview mit einem NGO-Sicherheitsexperten, 12. April 2012. 
nisationaler Ebene vorangetrieben und dabei auf verschiedene Art und Weise zur Professionalisierung des Sicherheitsmanagements beigetragen.

Auf Hauptquartier-Ebene versammeln die Netzwerke diejenigen Personen, die auf zentraler Ebene innerhalb einer NGO für Sicherheit zuständig sind. Dabei handelt es sich meist um NGO-Sicherheitsmanager. Diese Netzwerke bilden einerseits ein Forum, auf dem die Mitglieder sicherheitsrelevante Informationen austauschen können. Andererseits stellen sie auch bestimmte Produkte oder Dienstleistungen für ihre Mitglieder zur Verfügung (Alter/Hage 1993: 49), zum Beispiel Datenbanken oder Sicherheitsanalysen. Die Netzwerke werden von Mitgliedern der epistemischen Gemeinschaft initiiert, weiten sich im Laufe ihres Bestehens und meist mit dem Übergang von einem informellen zu einem formalisierten Netzwerk in der Regel aber über die epistemische Gemeinschaft hinaus aus. Dies hängt auch damit zusammen, dass die Mitgliedschaft in den formalisierten Netzwerken meist auf Organisationsbasis besteht, auch wenn die NGOs durch eine Person vertreten werden.

Zwar bedarf es für die Etablierung von solchen Netzwerken einer Reihe von Faktoren, z.B. ausreichend Ressourcen, aber die Interviewpartner waren sich einig, dass es vor allem auf das Engagement von Individuen ankommt. ${ }^{53}$ So entstand im Rahmen der bereits angesprochenen Trainings Ende der 1990er Jahre aus der epistemischen Gemeinschaft heraus mit der Security Advisory Group (SAG) des Verbandes InterAction die erste Form eines institutionalisierten NGO-Sicherheitsnetzwerks auf Hauptquartier-Ebene. Auf etwa fünfzehn Jahre später sind die Anfänge eines europäischen Pendants, des European Interagency Security Forums (EISF), ${ }^{54}$ zu datieren, als sich Vertreter einer Handvoll britischer NGOs, die auch zur epistemischen Gemeinschaft zu zählen sind, trafen, um auf ad-hoc-Basis über Sicherheitsthemen zu diskutieren. Nach einiger Zeit und einigen Treffen, die von den Beteiligten als sehr hilfreich bewertet wurden, entschied man sich, das Netzwerk auch auf europäische NGOs außerhalb Großbritanniens auszuweiten. ${ }^{55}$ Die Idee war es »to bring together like-minded [...] people working on the same issues «. ${ }^{56}$ Dabei sollte es nicht zur Duplikation bereits bestehender Strukturen kommen, sondern ein Sozialisationsprozess initiiert werden, der vertrauensbildend wirkt und dadurch den Austausch untereinander ermöglicht:

$»$ [to] fill a gap that we can see which is about bringing security advisers and managers together who are working on similar issues to have very free and frank discussions, to share information, to share policies and procedures, to learn from each other and to learn from each other's mistakes. And you know to do that you need to have quite [...] like a circle of trust [...] where you have the same people coming to the meetings and then you develop relationships and continuity and so on.$^{57}$

53 Siehe z.B. Interview mit einem NGO-Sicherheitsexperten 31. August 2011; 17. Januar 2012.

54 http://www.eisf.eu/, 27.07.2013.

55 Interview mit einem NGO-Sicherheitsexperten, 17. Januar 2012; Interview mit einem NGO-Sicherheitsexperten, 15. Dezember 2011; Interview mit einem NGO-Sicherheitsexperten, 07. Dezember 2011; Interview mit einem NGO-Sicherheitsexperten, 25. April 2012.

56 Interview mit einem NGO-Sicherheitsexperten, 17. Januar 2012.

57 Interview mit einem NGO-Sicherheitsexperten, 17. Januar 2012. 
Trafen sich die Sicherheitsmanager zunächst noch in ihrer Freizeit, so wurde schnell deutlich, dass ihnen die notwendigen Ressourcen fehlten, um zum Beispiel Informationen weiterzuleiten oder Datenanalysen durchzuführen. Daher beantragten sie Fördermittel bei Geberinstitutionen, die auch bewilligt wurden. ${ }^{58}$ EISF wurde dann 2008 institutionalisiert, zunächst mit der Ernennung eines Sicherheitsmanagers auf Teilzeitbasis und mit Erhalt einer Finanzierung des Department for International Development (DFID) im April 2009 durch die Einstellung von zwei Vollzeitkräften (EISF 2012). Derzeit (April 2014) hat EISF 58 Mitglieds-NGOs (EISF 2014a) und drei Mitarbeiter (EISF 2014b). Zusätzlich zu EISF wurden 2006 zwei weitere solche Netzwerke auf Hauptquartierebene etabliert. Eines davon ist das Dutch Security Network (DSN), ${ }^{59}$ welches bislang das einzige nationale Sicherheitsnetzwerk in Europa ist. Des Weiteren wurde im Rahmen der Action by Churches Together (ACT) Alliance, einem Dachverband von mehr als 130 Kirchen und kirchlichen Organisationen (ACT Alliance 2012a), eine Safety \& Security Community of Practice (SSCP, die zu Beginn Security Advisory Group und danach Global Working Group on Security hieß) gegründet. ${ }^{60}$

Auf Ebene der Einsatzgebiete ist das älteste und bekannteste institutionalisierte Netzwerk sicherlich das 2002 etablierte Afghanistan NGO Safety Office (ANSO) (Bickley 2006: 6). Andere Netzwerke existieren oder existierten zum Beispiel für Somalia (Somalia NGO Consortium 2012), Haiti, Gaza (Stoddard/Harmer 2010: 17) und Pakistan (PakSafe 2012). ${ }^{61}$ Auch die Sicherheitsnetzwerke auf Einsatzländerebene stellen eine Infrastruktur zur Verfügung, zum Beispiel in Form regelmäßiger Treffen oder Telefonlisten, die es den Mitglieds-NGOs ermöglichen, Informationen auszutauschen (Humanitarian Practice Network 2010: 17-19). Einige Netzwerke bieten auch Dienstleistungen oder Produkte an, zum Beispiel Sicherheitsanalysen, Beratung, Training, Krisenmanagement oder Liaison mit den Vereinten Nationen, Regierungen oder privaten Sicherheitsfirmen (Stoddard/Harmer 2010: 15-16).

Der Startschuss zur Etablierung von ANSO fand im Rahmen der Security Advisory Group (SAG) von InterAction durch Mitglieder der epistemischen Gemeinschaft statt. ${ }^{62}$ Die dort versammelten Sicherheitsmanager stimmten darin überein, dass es effektiver sei, wenn bestimmte Sicherheitsaufgaben, zum Beispiel Risikoanalysen, zentral durchgeführt werden, als wenn jede NGO diese für sich selbst durchführt. ${ }^{63}$ Sie betrieben Lobbyarbeit, um die in Afghanistan tätigen NGOs von der Idee zu überzeugen ${ }^{64}$ und darauf aufbauend leisteten etwa neun NGOs einen finanziellen Beitrag, um einen gemeinsamen Sicherheitsmanager zur Unterstützung ihrer Arbeit

58 Interview mit einem NGO-Sicherheitsexperten, 17. Januar 2012.

59 Interview mit einem NGO-Sicherheitsexperten, 15. Dezember 2011.

60 Interview mit einem NGO-Sicherheitsexperten, 15. Dezember 2011.

61 Für andere institutionalisierte Netzwerke siehe Bickley (2006: 13-15).

62 Interview mit einem NGO-Sicherheitsexperten, 01. Dezember 2009; Interview mit einem NGO-Sicherheitsexperten, 22. August 2011.

63 Interview mit einem NGO-Sicherheitsexperten, 22. August 2011.

64 Interview mit einem NGO-Sicherheitsexperten, 01. Dezember 2009. 
in Afghanistan einzustellen. ${ }^{65}$ Zudem beantragten diese NGOs Fördergelder für eine solche Stelle bei einzelnen Staaten und internationalen Organisationen. ${ }^{66}$ Die Gelder wurden bewilligt und ANSO wurde in Form eines formalen Sicherheitsnetzwerks etabliert.

Zusätzlich zu Sicherheitsnetzwerken auf Ebene der Hauptquartiere und Einsatzländer haben die Mitglieder der epistemischen Gemeinschaft einige nicht-profitorientierte Organisationen gegründet, die hauptsächlich Beratung und/oder Training anbieten und dabei auch zur Kooperation beitragen. Die Gründung der International NGO Safety and Security Association (INSSA) ${ }^{67}$ geht auf einige Mitglieder der epistemischen Gemeinschaft zurück, ${ }^{68}$ die folgende Probleme identifizierten: unterschiedliche Ansätze des Sicherheitsmanagements zwischen humanitären NGOs, wodurch die Kooperation unter den NGOs und eine effektive Reaktion auf die zunehmende Unsicherheit behindert wird (INSSA 2012b), eine fehlende Definition und Standardisierung einzelner Elemente eines Sicherheitsmanagements sowie damit einhergehend eine fehlende Struktur zur Professionalisierung der Sicherheitsmanager einerseits und zum Know-how-Transfer andererseits. ${ }^{69}$ Entsprechend liegt der Fokus von INSSA auf der Beratung und dem Training einzelner für Sicherheit zuständiger NGO-Mitarbeiter. Dabei erklärt INSSA ganz deutlich, dass die Standardisierung des Sicherheitsmanagements innerhalb der »NGO Community« ihr Ziel ist (INSSA 2013a; InterAction 2013). Die Organisation strebt einerseits einen Zertifizierungsprozess nach ISO-Standards für individuelle Mitarbeiter von NGOs an und andererseits ein Akkreditierungsverfahren einzelner Trainingskurse von unterschiedlichen Anbietern (INSSA 2013b). InterAction hat bereits 2006 Minimum Operating Security Standards verabschiedet, die von allen Mitglieds-NGOs befolgt werden müssen (InterAction 2006). ${ }^{70}$ Auch das Centre for Security and Development $(\mathrm{CSD})^{71}$ geht auf eine Initiative einiger Mitglieder der epistemischen Gemeinschaft zurück. Jene organisierten zunächst in ihrer Freizeit vor allem Trainings für NGOs, aber aufgrund der hohen Nachfrage wurde das CSD in Form einer nichtprofitorientieren Stiftung institutionalisiert. ${ }^{72}$ Derzeit bietet das CSD zum Beispiel einen Kurs zur Professionalisierung des organisationsinternen Sicherheitsmanagements (CSD 2013d) und mit Stay Safe einen Prozess an, um organisationsinternes Sicherheitsmanagement von NGOs entsprechend professioneller Standards zu etablieren (CSD 2013b). Darüber hinaus wurden auf Initiative einiger Mitglieder der epistemischen Gemeinschaft Beratungs- und Analyseeinrichtungen gegründet, die

65 Interview mit einem NGO-Sicherheitsexperten, 22. August 2011.

66 Interview mit einem NGO-Sicherheitsexperten, 22. August 2011.

67 http://ingossa.org/, 27.07.2013.

68 Interview mit einem NGO-Sicherheitsexperten, 26. Januar 2012.

69 Interview mit einem NGO-Sicherheitsexperten, 23. August 2011; Interview mit einem NGO-Sicherheitsexperten, 20. Oktober 2011.

70 Interview mit einem NGO-Sicherheitsexperten, 1.12.2009.

$71 \mathrm{http} / / / \mathrm{www}$. centreforsafety.org/, 27.07.2013.

72 Interview mit einem NGO-Sicherheitsexperten, 12. Oktober 2011. 
zum Beispiel Daten über die Unsicherheit humanitärer Helfer erheben und auswerten (Insecurity Insight) ${ }^{73}$ oder beratend tätig werden (Humanitarian Policy). ${ }^{74}$

Insgesamt ergibt sich ein dichtes Netz verschiedener Organisationen und Einrichtungen, deren Aufgaben und Portfolios sich ergänzen ${ }^{75}$ (siehe Tabelle 1) und die mit wissenschaftlichen Forschungseinrichtungen an der Tufts oder der Harvard Universität, staatlich finanzierten Einrichtungen zur Professionalisierung des Sicherheitsmanagement im humanitären Sektor, wie der Security Management Initiative (SMI), ${ }^{76}$ und anderen humanitären Akteuren wie den Vereinten Nationen und der EU kooperieren (können). ${ }^{77}$ Darüber hinaus werden viele der genannten Organisationen oder einzelne ihrer Projekte von internationalen Gebern finanziell unterstützt.

Tabelle 1: Ausgewählte NGO-Sicherheitskooperationen

\begin{tabular}{|l|l|l|l|}
\hline $\begin{array}{l}\text { Name und } \\
\text { Gründungsjahr }\end{array}$ & \multicolumn{1}{|c|}{ Ebene } & Mitglieder & \multicolumn{1}{c|}{ Dienstleistungen } \\
\hline $\begin{array}{l}\text { Security Advisory } \\
\text { Group (SAG) } \\
(1998 / 99)\end{array}$ & Hauptquartierebene & $\begin{array}{l}\text { US-amerikani- } \\
\text { sche NGOs }\end{array}$ & $\begin{array}{l}\text { Beratung, Information, Platt- } \\
\text { form für Austausch }\end{array}$ \\
\hline $\begin{array}{l}\text { European Interagency } \\
\text { Security Forum (EISF) } \\
\text { (2006) }\end{array}$ & Hauptquartierebene & $\begin{array}{l}\text { europäische } \\
\text { NGOs }\end{array}$ & $\begin{array}{l}\text { Information, advocacy, Liai- } \\
\text { son mit Gebern, Analysen, } \\
\text { good practices, Plattform für } \\
\text { Austausch }\end{array}$ \\
\hline $\begin{array}{l}\text { Dutch Security Net- } \\
\text { work (DSN) (2006) }\end{array}$ & Hauptquartierebene & $\begin{array}{l}\text { niederländische } \\
\text { NGOs }\end{array}$ & $\begin{array}{l}\text { Information, Plattform für } \\
\text { Austausch }\end{array}$ \\
\hline $\begin{array}{l}\text { Safety \& Security Com- } \\
\text { munity of Practice } \\
\text { (SSCP) der } \text { ACT Alli- } \\
\text { ance (2006) }\end{array}$ & Hauptquartierebene & $\begin{array}{l}\text { Mitglieds-NGOs } \\
\text { der ACT Allian- } \\
\text { ce }\end{array}$ & $\begin{array}{l}\text { Information, Handbücher, } \\
\text { Richtlinien, Trainings, Bera- } \\
\text { tung, Plattform für Austausch }\end{array}$ \\
\hline $\begin{array}{l}\text { Afghanistan NGO Safe- } \\
\text { ty Office (ANSO) } \\
\text { (2002) }\end{array}$ & Afghanistan & $\begin{array}{l}\text { in Afghanistan } \\
\text { aktive NGOs }\end{array}$ & $\begin{array}{l}\text { Beratung, Analyse, Informati- } \\
\text { on, Plattform für Austausch }\end{array}$ \\
\hline $\begin{array}{l}\text { Gaza NGO Safety Of- } \\
\text { fice (GANSO) (2008) }\end{array}$ & Gaza & $\begin{array}{l}\text { in Gaza aktive } \\
\text { NGOs }\end{array}$ & $\begin{array}{l}\text { Beratung, Analyse, Informati- } \\
\text { on, Plattform für Austausch }\end{array}$ \\
\hline
\end{tabular}

73 http://www.insecurityinsight.org/, 27.07.2013.

$74 \mathrm{http} / / / w w w . h u m a n i t a r i a n p o l i c y . o r g /, 27.07 .2013$.

75 Interview mit einem NGO-Sicherheitsexperten, 20. Oktober 2011.

$76 \mathrm{http} / / / \mathrm{www}$.securitymanagementinitiative.org/, 27.07.2013.

77 Interview mit einem NGO-Sicherheitsexperten, 23. August 2011.

78 Das Gründungsjahr wird nur angegeben, sofern es bekannt ist.

79 Das Spektrum der angebotenen Dienstleistungen kann sich über die Zeit verändern (s. z.B. Interview mit einem NGO-Sicherheitsexperten, 26. Januar 2012).

80 Seit Juli 2013 ist ANSO nicht mehr unabhängig, sondern ein von INSO geführtes Sicherheitsnetzwerk. 


\begin{tabular}{|l|l|l|l|}
\hline $\begin{array}{l}\text { Name und } \\
\text { Gründungsjahr }\end{array}$ & \multicolumn{1}{|c|}{ Ebene } & \multicolumn{1}{c|}{ Mitglieder } \\
\hline $\begin{array}{l}\text { NGO Safety Program } \\
\text { (NSP) for Somalia / } \\
\text { Somaliland (2004) }\end{array}$ & Somalia \\
\hline $\begin{array}{l}\text { PakSafe (2011) } \\
\text { Pakistan }\end{array}$ & $\begin{array}{l}\text { in Somalia akti- } \\
\text { ve NGOs }\end{array}$ & $\begin{array}{l}\text { Beratung, Analyse, Informati- } \\
\text { on, Training, Liaison mit loka- } \\
\text { len Akteuren und den VN, } \\
\text { Plattform für Austausch }\end{array}$ \\
\hline $\begin{array}{l}\text { International NGO } \\
\text { sociation (INSSA) } \\
\text { (2010) }\end{array}$ & $\begin{array}{l}\text { global, individuelle } \\
\text { Ebene }\end{array}$ & $\begin{array}{l}\text { individuelle Mit- } \\
\text { gliedschaft }\end{array}$ & $\begin{array}{l}\text { advocacy, Training, Standardi- } \\
\text { sierung, Plattform für Aus- } \\
\text { tausch }\end{array}$ \\
\hline $\begin{array}{l}\text { Center for Security and } \\
\text { Development (CSD) }\end{array}$ & $\begin{array}{l}\text { global; individuelle \& } \\
\text { Organitarian Fo- } \\
\text { rum (PHF) }\end{array}$ & $\begin{array}{l}\text { Beratung, Analyse, Informati- } \\
\text { on, Plattform für Austausch }\end{array}$ \\
\hline $\begin{array}{l}\text { Insecurity Insight } \\
\text { (2008) }\end{array}$ & $\begin{array}{l}\text { global; individuelle \& } \\
\text { Organisationsebene }\end{array}$ & $\begin{array}{l}\text { Information, Training, Bera- } \\
\text { tung, Analyse }\end{array}$ \\
\hline $\begin{array}{l}\text { Humanitarian Policy } \\
\text { global; individuelle \& } \\
\text { Organisationsebene }\end{array}$ & Analyse \\
\hline
\end{tabular}

Im Rahmen der genannten Netzwerke treibt die epistemische Gemeinschaft die Standardisierung des humanitären Sicherheitsmanagements voran und bedient sich dabei der Strategie der information politics. Ein Ergebnis der bereits angesprochenen Trainings Ende der 1990er Jahre war nicht nur die Etablierung der Security Advisory Group im Rahmen von InterAction, sondern auch die Publikation der Good Practice Review Nr. 8 Operational Security Management in Violent Environments von Koenraad van Brabant (2000), herausgegeben vom Humanitarian Practice Network des Overseas Development Institute in London, das als grundlegendes und bahnbrechendes Dokument für NGO-Sicherheitsmanagement gilt. ${ }^{82}$ Das Dokument wurde zwischenzeitlich unter Mitarbeit zahlreicher Mitglieder der epistemischen Gemeinschaft überarbeitet (Humanitarian Practice Network 2010b). Auch EISF publiziert regelmäßig Guidelines für humanitäre NGOs zu bestimmten Themen, wie zum Beispiel dem Umgang mit privaten Sicherheitsfirmen (Glaser 2011), dem Management von Entführungen (Buth 2010) oder der Integration von Gender-Mainstreaming in das Sicherheitsmanagement (Persaud 2012) und hat die Erarbeitung von Instrumenten zur Standardisierung zum Beispiel zur Berechnung der Kosten für Risikomanagement in Auftrag gegeben (Finucane 2013a; 2013b). Diese Dokumente wurden von den Interviewpartnern als sehr hilfreich bezeichnet. ${ }^{83}$

81 Dieses Netzwerk hieß ursprünglich NGO Security Preparedness and Support (NGO SPAS) Project for Somalia.

82 Interview mit einem NGO-Sicherheitsexperten, 23. August 2011.

83 Interviews mit NGO-Sicherheitsexperten, 09. September 2011, 20. Oktober 2011; 15. Dezember 2011; 26. Januar 2012. 
Die epistemische Gemeinschaft hat aber keine Entscheidungskompetenz, um innerhalb der NGOs Veränderungen zu bewirken, sondern sie kann nur beratend tätig werden; d.h. die von ihr formulierten Standards können nur als Vorschläge dienen, haben aber aus sich heraus keinen bindenden Charakter. Damit die von der epistemischen Gemeinschaft formulierten Standards angenommen werden, wird die Unterstützung einerseits derjenigen, die entsprechende Entscheidungen innerhalb von NGOs durchsetzen und Standards zum Sicherheitsmanagement etablieren, und andererseits derjenigen, die diese Standards dann umsetzen bzw. anwenden müssen, benötigt. Bei Ersteren handelt es sich vor allem um die Leitungsebene der NGOs und ggf. auch um Geber, die entsprechende Maßnahmen finanziell unterstützen können. Bei Letzteren handelt es sich um die einzelnen Mitarbeiter der NGOs. Entsprechend muss die epistemische Gemeinschaft von den genannten Akteuren als legitime Autorität anerkannt werden. Hierzu ist es wichtig, dass die epistemische Gemeinschaft den »worldview« (Davis Cross 2013: 147) derjenigen Akteure berücksichtigt, die die von ihr formulierten Standards etablieren und umsetzen sollen bzw. auf deren system of knowledge (Antoniades 2006: 22-23) eingeht, um Akzeptanz für ihre Vorschläge zu erlangen.

Dabei kommen der epistemischen Gemeinschaft mindestens zwei Entwicklungen zugute. Dies ist zum einen der grundsätzliche Trend zur Professionalisierung und Standardisierung der humanitären Hilfe, sowohl bezüglich der Arbeit der Organisationen als auch der einzelnen Mitarbeiter. Beispiele für Professionalisierung und Standardisierung auf Organisationsebene sind der »Verhaltenskodex der internationalen Rotkreuz- und Rothalbmondbewegung sowie von NGOs in der Katastrophenhilfe« (IFRC 2013) sowie das 1997 gegründete SPHERE-Projekt, welches die Humanitarian Charter and Minimum Standards in Humanitarian Response (SPHERE 2011) herausgibt, die weltweit anerkannt sind. Zur Professionalisierung einzelner humanitärer Helfer hat sich zum Beispiel 2010 die International Association of Professionals in Humanitarian Assistance and Protection (PHAP) gegründet, deren Ziel es ist, "to respond to a growing demand for professional development and networking opportunities among individuals deployed in the context of humanitarian crises « (PHAP 2013). Zum anderen haben humanitäre NGOs ihren Mitarbeitern gegenüber eine Sorgfaltspflicht mit Blick auf ihre Sicherheit (Act Alliance 2011; ECHO 2004), bei deren Missachtung Versicherungen unter Umständen nicht für die entstandenen Kosten aufkommen und Mitarbeiter ihre Organisation möglicherweise verklagen können (Kemp/Merkelbach 2011). Dies nutzt die epistemische Gemeinschaft im Sinne von »accountability politics« (Keck/Sikkink 1998: 24-25) als Argument, um humanitäre NGOs von der Etablierung eines Sicherheitsmanagements zu überzeugen (Kemp/Merkelbach 2011; Van Brabant 2010: 6; Finucane 2013a: 3).

Des Weiteren trägt die epistemische Gemeinschaft zu Paradigmenwechseln innerhalb der »humanitären Community« bei, und zwar in dem Sinne, dass sie die Rolle von Sicherheit für humanitäre NGOs und ihren Umgang damit anders konzipiert, als es bisher üblicherweise der Fall war. Hierzu rahmt die epistemische Gemeinschaft den Zusammenhang zwischen humanitären Prinzipien und Sicherheit 
grundsätzlich neu. Anstelle des Arguments, dass sich humanitäre NGOs aufgrund ihrer Identität als neutrale, unabhängige und unparteiliche Organisationen nicht um ihre Sicherheit kümmern müssten, basiert der Diskurs der epistemischen Gemeinschaft der humanitären Sicherheitsexperten auf der Annahme, dass humanitäre NGOs gerade aufgrund ihrer Identität als neutrale, unabhängige und unparteiliche Organisationen eine besondere Art von Sicherheitsmanagement benötigen. Hier kommt der epistemischen Gemeinschaft zugute, dass sie zusätzlich zur technischen Expertise auch das system of knowledge (Antoniades 2006) derjenigen versteht, an die sich der Diskurs richtet und sie ihren Diskurs entsprechend ausrichten kann. Ein Paradigmenwechsel betrifft das Sicherheitsverständnis. Basierend auf ihrer humanitären Identität und ihrer »activist culture« (Edwards 1997: 70) liegt der Fokus der Arbeit der NGOs auf der Durchführung humanitärer Programme zur Linderung akuter Not (Van Brabant 2001a: 189). Entsprechend werden Sicherheitsmaßnahmen traditionell als eine Behinderung für die Durchführung humanitärer Programme angesehen. ${ }^{84}$ Die epistemische Gemeinschaft der Sicherheitsexperten versucht mittels der Sicherheitsnetzwerke die Ansicht zu verbreiten, dass Sicherheitsmaßnahmen die Durchführung humanitärer Programme nicht behindern, sondern - im Gegenteil - erst ermöglichen: »Safety and security is not an end in itself but a condition for sustainable access, and is thereby an enabler to aid agencies' institutional and operational goals of presence and program implementation « (Merkelbach/ Daudin 2011: 1; siehe auch Humanitarian Practice Network 2010b: 7). ${ }^{85}$ So erklärte ein Interviewpartner:

»...the program managers are always worried of saying, if they include security they are going to be told >no, you can't do it or >no, you can't go there understand that security is going to tell you where you can go, but this is how you are going to have to do it in order to be safe.$^{86}$

Auf Basis der geteilten Grundannahme, dass ein passiver Umgang mit Sicherheit nicht mehr ausreicht, fordert die epistemische Gemeinschaft eine aktive Auseinandersetzung jeder NGO mit ihrer Sicherheit (Fast/O'Neill 2010: 5) und ein Überdenken der grundlegenden Prinzipien. Davon ausgehend, dass NGOs »[are] mainly relying on community acceptance $[\ldots] \ll,{ }^{87}$ schlägt die epistemische Gemeinschaft dafür einen aktiven Akzeptanzansatz vor, der von den NGOs verlangt, »[to] deliberately work [...] to gain and sustain consent from all stakeholders « (Fast/O'Neill 2010: 5). Alternative Sicherheitsansätze, wie derjenige der Vereinten Nationen, wurden von den Interviewpartnern als inkompatibel mit dem der NGOs bezeichnet. Die Vereinten Nationen zum Beispiel würden sich »einbunkern«, ${ }^{88}$ was nicht nur dem unter NGOs verbreiteten Akzeptanz-Ansatz (Martin 1999: 5-6) widerspricht, sondern auch diejenigen Meinungen unterstützt, denen zufolge Sicherheitsmaßnah-

84 Interview mit einem NGO-Sicherheitsexperten, 09. September 2011.

85 Interview mit einem NGO-Sicherheitsexperten, 22. August 2011.

86 Interview mit einem NGO-Sicherheitsexperten, 09. September 2011.

87 Interview mit einem NGO-Sicherheitsexperten, 17. November 2011.

88 Interview mit einem NGO-Sicherheitsexperten, 10. Oktober 2011. 
men die Durchführung humanitärer Programme verhindern, was den Bemühungen der epistemischen Gemeinschaft entgegensteht. ${ }^{89}$

Aus dem geteilten Verständnis der Interdependenz ergibt sich für die Rolle von Sicherheit und das Sicherheitsmanagement humanitärer NGOs aus Sicht der epistemischen Gemeinschaft »the absolute need to share information and cooperate on an inter-agency level $\ll,{ }^{90}$ wobei einhellig davon ausgegangen wird, dass die Kooperation in Sicherheitsfragen für alle Beteiligten von Nutzen ist (Stoddard/Harmer 2010: 14). Dies führt zu einem Paradigmenwechsel mit Blick auf den Umgang mit Informationen von einem »need to know « zu einem »need to share «. ${ }^{11}$ Ersteres ist ein Grundsatz aus dem Bereich der Geheimdienstarbeit, dessen Verankerung in der humanitären Hilfe damit zu tun hat, dass einerseits Sicherheitsfragen lange Zeit als nicht genuin humanitäres Thema verstanden wurden und andererseits die erste Generation der Sicherheitsmanager großteils aus ehemaligen Angehörigen staatlicher Sicherheitskräfte bestand. Doch aufgrund der Interdependenz ist es unmöglich zu wissen, welche Information für andere hilfreich oder gar notwendig ist. ${ }^{92}$ Daher versuchen die Mitglieder der epistemischen Gemeinschaft, das need to share-Prinzip innerhalb der »humanitären Community« zu etablieren, damit alle die Möglichkeit haben, informierte Entscheidungen zu treffen. Das Centre for Safety and Development (CSD) erklärt zum Beispiel: »We believe that sharing security knowledge and skills enables other humanitarian organisations to work in unsafe areas in order to reduce human suffering and bring about a better world« (CSD 2012a). Und ein Interviewpartner erklärte, dass seine Organisation auf Anraten eines externen Beraters nun Mitglied bei EISF sei. ${ }^{93}$

Um entsprechende Paradigmenwechsel durchzusetzen, bedarf es Personen, die das NGO-mindset verinnerlicht haben und den worldview und die knowledge structures derjenigen, die die Standards etablieren und umsetzen sollen, teilen. Wenn solche Prozesse von nicht-Mitgliedern der epistemischen Gemeinschaft initiiert werden, werden sie von der »humanitären NGO-Community« nicht oder vergleichsweise schlecht angenommen. So ist das Konzept Saving Lives Together (SLT), das die Kooperation in Sicherheitsfragen zwischen den Vereinten Nationen (VN) und NGOs regeln soll, vielen NGO-Mitarbeitern nicht bekannt, und folglich wird es kaum umgesetzt (Micheni/Kuhanendran 2010: 4). Zudem ist die Beteiligung von NGO-Mitarbeitern an Besprechungen zur Sicherheitslage, die von den VN organisiert werden, geringer als an entsprechenden Treffen, die von den NGOs selbst durchgeführt werden (Micheni/Kuhanendran 2010: 6-7). Des Weiteren scheiterte zum Beispiel ein Vorschlag einer privaten Sicherheitsfirma, einen Kooperationsmechanismus für NGOs zu Sicherheitsthemen zu etablieren, und auch Sicherheitsnetzwerke, bei denen militärische Akteure involviert sind, werden von NGOs nicht angenommen (Renouf 2011: 154). Nach Ansicht eines Experten wird daran

89 Interview mit einem NGO-Sicherheitsexperten, 10. Oktober 2011.

90 Interview mit einem NGO-Sicherheitsexperten, 13. Januar 2012.

91 Interview mit einem NGO-Sicherheitsexperten, 29. August 2012.

92 Interview mit einem NGO-Sicherheitsexperten, 29. August 2012.

93 Interview mit einem NGO-Sicherheitsexperten, 18.03.2010. 
deutlich, dass »any outsider attempting to contribute to humanitarian security networks must be seen as legitimate by insiders, and abide by aid agencies' codes and cultural references« (Renouf 2011: 155).

Einige NGO-Sicherheitsnetzwerke hatten zunächst Sicherheitsmanager »with a strong military perspective« eingestellt, die zwar das technische Know-how hatten, »but they didn't have the NGO mindset, the proper understanding of the aid community. So that had definitely not helped in bringing confidence among the NGO community and willingness to share. So [...] that was a mistake which was commonly repeated «. ${ }^{94}$ Grundsätzlich hat eine (wahrgenommene) Nähe von NGO-Sicherheitsnetzwerken zu staatlichen Sicherheitsakteuren in einigen Fällen zur Abspaltung und/oder Gründung separater NGO-Sicherheitsnetzwerke geführt, die den Erwartungen und Anforderungen der beteiligten NGOs besser entsprachen. ${ }^{95}$ Als Konsequenz haben NGO-Sicherheitsnetzwerke ihr Personal ausgetauscht und Mitarbeiter eingestellt, die das $N G O$ mindset verinnerlicht haben und die dadurch nicht nur von der epistemischen Gemeinschaft, sondern auch der »NGO-Community« im Allgemeinen anerkannt werden. ${ }^{96}$ Daran wird deutlich, dass die gemeinsame Identität der Mitglieder der epistemischen Gemeinschaft nicht nur für den internen $\mathrm{Zu}$ sammenhalt der Gemeinschaft notwendig ist, sondern dieser auch nach außen legitime Autorität verschafft. Entsprechend ist es für den Erfolg einer epistemischen Gemeinschaft wichtig, dass sowohl unter ihren Mitgliedern als auch zwischen diesen und den von ihrer Standardsetzung betroffenen Akteuren »a shared worldview» (Davis Cross 2013: 147) existiert (Antoniades 2006).

Der epistemischen Gemeinschaft gelingt es dabei auch aufgrund ihrer heterogenen Zusammensetzung bei unterschiedlichen Akteuren Gehör zu finden und als legitime Autorität anerkannt zu werden. Dabei geht es nicht nur darum, Strukturen des Sicherheitsmanagements formal niederzuschreiben, sondern ein solches Sicherheitsmanagement auch auf allen Ebenen einer NGO im Rahmen eines kohärenten Systems umzusetzen. Wie dieses genau ausgestaltet ist, ist aus Sicht der epistemischen Gemeinschaft nebensächlich:

»But at least there is a system in place, this is what it is important. What is dangerous is when there is no clear responsibility [...] and people may [have] got [some] kind of paper somewhere and they think syou see, we do security<. But it's not about paper, it's about do you have responsible persons who understand that it is their main responsibility, who understand that their job is on the line if something goes wrong, so that they take things seriously and they $[\ldots]$ [pay] attention $[\ldots][$ to $]$ security $\ll{ }^{97}$

Dazu versuchen die Mitglieder, alle betroffenen Stakeholder innerhalb und außerhalb der NGOs zu informieren (information politics). Eines von INSSA's Zielen ist es daher, »to help inform policymakers, government agencies, donor organizati-

94 Interview mit einem NGO-Sicherheitsexperten, 25. April 2012; siehe auch Interview mit einem NGO-Sicherheitsexperten, 09. September 2011; 17. November 2011.

95 Interview mit einem NGO-Sicherheitsexperten, 25. April 2012; Interview mit einem NGO-Sicherheitsexperten, 04. November 2011.

96 Interview mit einem NGO-Sicherheitsexperten, 04. November 2011; Interview mit einem NGO-Sicherheitsexperten, 17. November 2011.

97 Interview mit einem NGO-Sicherheitsexperten, 17. November 2011. 
ons, and the general public on the risks faced by aid workers and build better support mechanisms to ensure their safety and security" (INSSA 2012a). Die Aussage eines Vertreters eines Netzwerks macht deutlich, dass dabei Konkurrenz und Wettbewerb in den Hintergrund treten:

$»[\ldots]$ it's also my task [...] to go to them and convince them that they should take security more seriously. And they can organise security courses together with us, they can go to other organisations, we basically don't care as long as they make sure to have security on the agenda.$^{98}$

Zu diesem Zwecke organisierten zum Beispiel CSD (2012b) sowie die ACT Alliance Security Working Group (ACT Alliance 2012b) jeweils »Sicherheitswochen « ${ }^{99}$ Dabei geht es teilweise darum, »dicke Bretter zu bohren«, indem die Mitglieder der epistemischen Gemeinschaft bei jeder Veranstaltung wiederholen, wie wichtig professionelles Sicherheitsmanagement ist. ${ }^{100}$

Denn auch wenn viele NGOs inzwischen ein Sicherheitsmanagement etabliert haben, so fehlt es oftmals an einer Sicherheitskultur, und Sicherheitsmanager werden vom Management nur bedingt unterstützt und fühlen sich von diesem teilweise »ignoriert« (Van Brabant 2010: 5). Daher laden einzelne Netzwerke NGO-Direktoren, aber auch Mitarbeiter von NGOs, die auf den ersten Blick nichts mit Sicherheit zu tun haben, zu ausgewählten Veranstaltungen ein, »trying to reach out within the organisations as well, recognising the different [...] areas that also have an impact on security «. ${ }^{101}$ Dabei kommen der epistemischen Gemeinschaft sowohl ihr technisches Know-how als auch ihre Kenntnisse über das NGO mindset zugute. Sicherheitsnetzwerke identifizieren teilweise ganz gezielt organisationsinterne Hindernisse für Sicherheitsmanagement und versuchen diese auszuräumen. So wurde bei einer Veranstaltung von EISF das Problem erkannt, dass NGOs und Geber unterschiedliche Annahmen über die Bereitschaft der Geber zur Finanzierung von Sicherheitsmaßnahmen haben. Während die für die Formulierung entsprechender Anträge in den NGOs zuständigen Mitarbeiter der Ansicht waren, dass Geber keine Sicherheitsmaßnahmen finanzieren, erklärten die Geber-Vertreter das Gegenteil. Daher entschied sich das Netzwerk, eine entsprechende Handreichung für die NGOs zu erstellen, die sich direkt an die für Projektanträge zuständigen Mitarbeiter richtet (Finucane 2013a; 2013b). ${ }^{102}$

Der epistemischen Gemeinschaft gelingt es auch über die NGOs hinaus, Gehör für ihr Anliegen zu finden. Irish Aid, die Entwicklungshilfe-Abteilung der irischen Regierung, hat Mitglieder der epistemischen Gemeinschaft damit beauftragt, Guidelines for NGO Professional Safety \& Security Risk Management (Irish Aid 2013) zu formulieren, wodurch diejenigen NGOs, die von Irish Aid Gelder erhalten, auf-

\footnotetext{
98 Interview mit einem NGO-Sicherheitsexperten, 12. Oktober 2011.

99 Interview mit einem NGO-Sicherheitsexperten, 15. Dezember 2011.

100 Interview mit einem NGO-Sicherheitsexperten, 31. August 2011.

101 Interview mit einem NGO-Sicherheitsexperten, 07. Dezember 2011; siehe auch Interview mit einem NGO-Sicherheitsexperten, 15. Dezember 2011.

102 Interview mit einem NGO-Sicherheitsexperten, 07. Dezember 2011.
} 
gefordert werden, ein Sicherheitsmanagement zu etablieren. ${ }^{103}$ Nach Aussage eines Experten möchte die irische Regierung in diesem Zusammenhang eine Vorreiterrolle spielen, und die Standards sind so angelegt, dass andere Regierungen dem irischen Beispiel folgen und diese übernehmen können. ${ }^{104}$ Somit geht die Standardisierung inzwischen über die Selbstregulierung hinaus.

Die epistemische Gemeinschaft ist jedoch bestrebt, dass es nicht nur zu Anpassungsprozessen kommt. Auch wenn einige NGOs nur deshalb Sicherheitspolitiken und entsprechende Strukturen und Verfahren etablieren, weil Geber oder Versicherungen dies von ihnen verlangen, damit die Organisation im Falle eines Sicherheitsvorfalles nicht haftbar gemacht werden kann, ${ }^{105}$ geht es der epistemischen Gemeinschaft darüber hinaus um ein Mainstreaming von Sicherheit innerhalb der NGOs, wobei Elemente eines Sicherheitsmanagements auf allen Ebenen etabliert werden sollen: »[Sicherheitsmanagement] is not an add-on or a luxury« (Humanitarian Practice Network 2010b: 3). So erklärt das Centre for Security and Development (CSD): »Our activities are not a tick-in-the-box exercise, we believe in making a difference for aid workers and NGOs alike« (CSD 2013a). Entsprechend bietet CSD Prozesse an, bei denen es darum geht, Sicherheit in die gesamte Organisation und in die hearts and minds der Mitarbeiter zu integrieren (CSD 2013c). Auch einzelne NGOs sind bemüht, das Sicherheitsmanagement in ihren Organisationen auf eine möglichst breite Basis zu stellen. So werden in mindestens zwei Organisationen die Prozesse und Strukturen des organisationsinternen Sicherheitsmanagements nicht von oben, sondern durch die jeweils betroffenen Mitarbeiter in den einzelnen Regionalbüros selbst etabliert. ${ }^{106}$ Die Institutionalisierung hilft auch einzelnen NGO-Sicherheitsmanagern, Sicherheit auf die Agenda ihrer jeweiligen Organisation zu setzen und innerhalb ihrer NGO mehr Einfluss zu erhalten (Daudigeos 2013: 735). Mit Blick auf EISF erklärte ein Interviewpartner:

»The security managers or directors got more self-confidence because they could rely on networks, so they were better informed, they could compare their organisation's level of security management or awareness [...] to other organisations and bring this back to their organisation, to their head of organisation, to their board, and this could give them solid [...] argument to invest in security ${ }^{107}$

Die einzelnen Prozesse der Institutionalisierung, der Standardisierung und des Paradigmenwechsels können sich gegenseitig verstärken. Die Erfahrungen, die humanitäre NGOs und ihre Mitarbeiter mit institutionalisierten Kooperationen wie ANSO machen, einerseits und die zunehmende Schaffung von Sicherheitsmanagerpositionen innerhalb von NGOs durch die Professionalisierung des Sicherheitsmanagements andererseits haben laut Aussage der Interviewpartner das Verständnis für die Notwendigkeit und den Mehrwert von Sicherheitskooperation innerhalb der

103 Interview mit einem NGO-Sicherheitsexperten, 20. Oktober 2011.

104 Interview mit einem NGO-Sicherheitsexperten, 20. Oktober 2011; Interview mit einem NGO-Sicherheitsexperten, 12. April 2012.

105 Interview mit einem NGO-Sicherheitsexperten, 26. Januar 2012.

106 Interview mit einem NGO-Sicherheitsexperten, 18.03.2010; Interview mit einem NGOSicherheitsexperten, 16.11.2009.

107 Interview mit einem NGO-Sicherheitsexperten, 25. April 2012. 
»NGO-Community« gefördert und ein gewisses politisches Momentum geschaffen, das die Etablierung von weiteren Sicherheitsnetzwerken, wie dem European Interagency Security Forum (EISF), ${ }^{108}$ und der Safety \& Security Community of Practice der ACT Alliance ${ }^{109}$ ermöglichte.

\section{Fazit}

Humanitäre NGOs sind seit einigen Jahren mit einer zunehmenden Unsicherheit in einigen ihrer Einsatzgebiete konfrontiert. Dies stellt die NGOs vor eine doppelte Herausforderung. Zum einen sind ihre Mitarbeiter und damit ihre Programme gefährdet. Zum anderen müssen humanitäre NGOs einsehen, dass die Durchführung humanitärer Hilfe auf Basis der Prinzipien Neutralität, Unparteilichkeit und Unabhängigkeit allein keinen (ausreichenden) Schutz für ihre Mitarbeiter und ihre Programme bietet. Somit stellt die physische Unsicherheit die Identität humanitärer NGOs in Frage. Daher fällt es humanitären NGOs schwer, sich aktiv mit ihrer Sicherheit auseinanderzusetzen, grundlegende Prinzipien zu überdenken und ein aktives Sicherheitsmanagement zu etablieren, gleichwohl dies überlebenswichtig sein kann. Trotz entsprechender organisationsinterner Hindernisse haben immer mehr humanitäre NGOs in den letzten Jahren ein internes Sicherheitsmanagement etabliert und kooperieren vermehrt untereinander in Sicherheitsfragen.

Dies wird hier mit dem Engagement der epistemischen Gemeinschaft humanitärer Sicherheitsexperten erklärt. Dieser gelang es, durch die Institutionalisierung von Sicherheitskooperationen sowie durch eine neue Rahmung des Zusammenhangs zwischen humanitären Prinzipien und Sicherheit und damit einhergehenden Paradigmenwechseln, organisationsinterne Hindernisse zu überwinden und NGOs von der Notwendigkeit eines professionellen organisationsinternen und -übergreifenden Sicherheitsmanagements zu überzeugen. Von der epistemischen Gemeinschaft etablierte Standards werden von vielen NGOs übernommen und inzwischen auch im Falle Irlands von einer Regierung. Dabei ist die epistemische Gemeinschaft bestrebt, die »humanitäre Community« davon zu überzeugen, dass Sicherheitsmanagement nicht aus formalen Gründen etabliert werden sollte, um Schadensansprüche von Mitarbeitern abzuwehren, sondern dass NGOs einerseits ihre grundlegenden Prinzipien überdenken und andererseits Sicherheit innerhalb der Organisation auf allen Ebenen integrieren müssen, weil Sicherheit die notwendige Voraussetzung für die Durchführung humanitärer Programme ist.

Die epistemische Gemeinschaft ist erfolgreich aufgrund ihres homogenen professionellen Selbstverständnisses, das nach innen einen hohen Grad an Zusammenhalt bewirkt und ihr nach außen legitime Autorität verschafft. Dieses professionelle Selbstverständnis, die Identität der Mitglieder der epistemischen Gemeinschaft als

108 Interview mit einem NGO-Sicherheitsexperten, 25. April 2012; siehe auch Interview mit einem NGO-Sicherheitsexperten, 26. Januar 2012.

109 Interview mit einem NGO-Sicherheitsexperten, 13. Januar 2012. 
humanitäre Sicherheitsexperten und ein damit einhergehender shared worldview beruhen einerseits auf ihrer fachlichen Expertise und andererseits auf einer geteilten Wertebasis, die im Rahmen einer gemeinsamen Sozialisation und geteilter Wissensbestände vermittelt werden und zum Zusammenhalt der Mitglieder der epistemischen Gemeinschaft führen. Die Kombination der genannten Faktoren bewirkt, dass sich die Mitglieder der »humanitären Community« mit den von der epistemischen Gemeinschaft etablierten Standards identifizieren können.

Durch den vorliegenden Aufsatz wurde erstens ein Beitrag zur Wiederbelebung des Konzeptes der epistemischen Gemeinschaft im Sinne einer Neuausrichtung geleistet (Davis Cross 2013). Das hier dargestellte Verständnis einer epistemischen Gemeinschaft ermöglicht es, Koalitionen aus Individuen aus unterschiedlichsten Bereichen und Sektoren als politische Akteure zu verstehen, klar zu benennen, wer $\mathrm{zu}$ einer epistemischen Gemeinschaft gehört und wer nicht, sowie den Einfluss einer epistemischen Gemeinschaft zu erklären. Dabei wird ein bisher vernachlässigter, aber in Zeiten von Drehtüreffekten und individueller Karriereverläufe, die nicht mehr an einen bestimmten Arbeitgeber oder einen bestimmten Sektor gebunden sind, einflussreicher transnationaler politischer Akteur in den Fokus der Analyse gerückt. Des Weiteren wird die Bedeutung nichtstaatlicher Akteure sowohl als Regelsetzer als auch als Regelempfänger betont und es werden Kriterien dafür formuliert, dass nichtstaatliche Akteure von anderen nichtstaatlichen Akteuren gesetzte Standards akzeptieren.

Damit leistet der Aufsatz - zweitens - einen Beitrag zur Diskussion über die für den Einfluss transnationaler Akteure entscheidenden Faktoren. Indem der Aufsatz nicht nur untersucht, wie eine epistemische Gemeinschaft entsteht und welche Strategien sie anwendet, sondern auch was sie intern zusammenhält, trägt er einerseits neueren Arbeiten Rechnung, die zu dem Ergebnis kommen, dass die Binnenverfasstheit transnationaler Akteure deren Einfluss bestimmt (z.B. Wong 2012). Dabei gelingt es aufbauend auf der Argumentation von Davis Cross (2013) Faktoren zu identifizieren, die intern Zusammenhalt herstellen und nach außen hin Autorität gewährleisten. Andererseits erweitert der Aufsatz das entsprechende Spektrum zu untersuchender Faktoren um den des Vertrauens. Dabei handelt es sich zwar um keinen Newcomer in den Internationalen Beziehungen im Allgemeinen (z.B. Rathbun 2011a; 2011b) und mit Blick auf humanitäre Hilfe im Speziellen (Saab et al. 2013; Stephenson 2005), doch bisherige Arbeiten zu epistemischen Gemeinschaften haben das Vertrauen externer Akteure in diese Gemeinschaften (Adler 1992; Haas 1992b) und nicht innerhalb dieser Gemeinschaften thematisiert. Daher wissen wir immer noch wenig über die spezifische Form des Vertrauens, das innerhalb epistemischer Gemeinschaften von Bedeutung ist. Mit Blick auf die aktuelle Debatte über Vertrauen in den Internationalen Beziehungen (Kaina 2011; Levi/Murphy 2006; Rathbun 2011a; 2011b) könnten entsprechende Analysen nicht nur für die Diskussion über epistemische Gemeinschaften, sondern auch für die Funktionsweise anderer transnationaler Akteure und Prozesse der Standardsetzung relevant sein.

Auch hinsichtlich der von ihnen vorangetriebenen Standardisierung wird deutlich, dass epistemische Gemeinschaften politische Akteure sind, die zu transnatio- 
naler governance beitragen. Die Standardisierungstendenzen, vor allem die Standardisierung von Trainings im Sinne von Ausbildung und Wissensvermittlung, können als Ausdruck einer Rationalisierung verstanden werden (Ritzer 1975: 629; aufbauend auf Max Weber 2005: 583-587). Dadurch werden bestimmte Formen des Trainings und damit des Sicherheitsmanagements mit einem Gütesiegel versehen und somit als gute Formen des Umgangs mit Sicherheit klassifiziert. Dabei hat die Standardsetzung der epistemischen Gemeinschaft eine transnationale Ordnungsfunktion. Diese Standardsetzung kann jedoch wiederum, insbesondere wenn sie von staatlicher Seite gefördert wird (s. Beispiel Irish Aid), die Identität humanitärer NGOs als neutrale, unabhängige und unparteiliche Akteure beeinträchtigen.

\section{Literatur}

Abrahamsen, Rita/Williams, Michael C. 2011: Security Beyond the State: Private Security in International Politics, Cambridge, MA.

ACT Alliance 2011: ACT Staff Safety and Security Guidelines. A Handbook for ACT Staff, in: http://www.actalliance.org/resources/policies-and-guidelines/security/ ACT_Saff_Safety_-_Security_Guidelines_vers_2011.pdf; 27.07.2013.

ACT Alliance 2012a: ACT 2012: Home, in: http://www.actalliance.org/; 9.8.2012.

ACT Alliance 2012b: ACT Safety and Security Week, in: http://www.actalliance.org/stories/ safety-and-security-week; 14.8.2012.

Adler, Emanuel 1992: The Emergence of Cooperation: National Epistemic Communities and the International Evolution of the Idea of Nuclear Arms Control, in: International Organization 46: 1, 101-145.

Alter, Catherine/Hage, Jerald 1993: Organizations Working Together, Newbury Park, CA.

Antoniades, Andreas 2006: Epistemic Communities, Epistemes and the Construction of (World) Politics, in: Global Society 17: 1, 21-38.

Argyris, Chris/Schön, Donald A. 2007: Theory in Practice: Increasing Professional Effectiveness, San Francisco, CA.

Arts, Bas 2006: Non-state Actors in Global Environmental Governance: New Arrangements Beyond the State, in: Koenig-Archibugi, Mathias/Zürn, Michael (Hrsg.): New Modes of Governance in the Global System: Exploring Publicness, Delegation and Inclusiveness. Basingstoke, 177-199.

Barnett, Michael 2009: Evolution Without Progress? Humanitarianism in a World of Hurt, in: International Organization 63: 4, 621-663.

Barnett, Michael/Weiss, Thomas G. 2008a (Hrsg.): Humanitarianism in Question. Politics, Power, Ethics. Ithaca, NY.

Barnett, Michael/Weiss, Thomas G. 2008b: Humanitarianism. A Brief History of the Present, in: Barnett, Michael/Weiss, Thomas G. (Hrsg.): Humanitarianism in Question. Politics, Power, Ethics. Ithaca, NY, 1-48.

Barnett, Michael/Snyder, Jack 2008: The Grand Strategies of Humanitarianism, in: Barnett, Michael/Weiss, Thomas G. (Hrsg.): Humanitarianism in Question. Politics, Power, Ethics. Ithaca, NY, 143-171.

Bickley, Shaun 2006: NGO Security Collaboration Guide, Brussels.

Börzel, Tanja/Risse, Thomas 2005: Public-Private Partnerships: Effective and Legitimate Tools of Transnational Governance? in: Grande, Edgar/Pauly, Louis W. (Hrsg.): Complex Sovereignty: Reconstructing Political Authority in the Twenty-first Century. Toronto, 195-216. 
Bollettino, Vincenzo 2008: Understanding the Security Management Practices of Humanitarian Organisations, in: Disasters 32: 2, 263-279.

Bronner, Uta 2003: Humanitäre Helfer in Krisengebieten. Motivation, Einsatzerleben, Konsequenzen - Eine psychologische Analyse, Münster.

Buth, Pete 2010: Abduction Management: EISF Briefing Paper, London.

Cooley, Alexander/Ron, James 2002: The NGO Scramble: Organizational Insecurity and the Political Economy of Transnational Action, in: International Security 27: 1, 5-39.

CSD 2012a: Why we do it, in: http://www.centreforsafety.org/why-we-do-it, 06.08.2012.

CSD 2012b: NGO Security Week 2011, in: http://www.centreforsafety.org/nieuwsindex/ N3N39/CFS/NGO-Security-Week-2011, 10.08.2012.

CSD 2013a: How We Work, in: http://www.centreforsafety.org/how-we-work, 23.07.2013.

CSD 2013b: Stay Safe, in: http://www.centreforsafety.org/Stay-safe, 23.07.2013.

CSD 2013c: Stay Safe Partnership, in: http://www.centreforsafety.org/content/CSD_Security_Packages_v6_pdf, 23.07.2013.

CSD 2013d: HQ Security Management Course, in: http://www.centreforsafety.org/content/ Downloads/HQ_Security_Management_Course_USA_2012.pdf, 23.07.2013.

Daudigeos, Thibault 2013: In Their Profession's Service: How Staff Professionals Exert Influence in Their Organization, in: Journal of Management Studies 50: 5, 722-749.

Davis Cross, Mai'a K. 2013: Rethinking Epistemic Communities Twenty Years Later, in: Review of International Studies 39: 1, 137-160.

Dingwerth, Klaus 2005: The Democratic Legitimacy of Public-Private Rule Making: What Can We Learn from the World Commission on Dams? in: Global Governance 11: 1, 65-84.

Donini, Antonio 1996: Auf der Welle reiten, bis sie bricht. Intervention und der Süden, in: Debiel, Tobias/Nuscheler, Franz (Hrsg.), Der neue Interventionismus: Humanitäre Einmischung zwischen Anspruch und Wirklichkeit, Bonn, 185-206.

Drake, William J./Nicolaïdis, Kalypso 1992: Ideas, Interests, and Institutionalization: "Trade in Services" and the Uruguay Round, in: International Organization 46: 1, 37-100.

DRK 2013: Die Grundsätze des Roten Kreuzes und Roten Halbmondes, in: http://www.drk.de/ ueber-uns/auftrag/grundsaetze.html, 24.01.2013.

Eberwein, Wolf-Dieter 2004: Humanitäre Hilfe - Krieg und Terror. Kontinuität und Wandel eines Politikfeldes, in: http://edoc.vifapol.de/opus/volltexte/2009/1182/pdf/p04_301.pdf, 09.04.2014.

Ebrahim, Alnoor 2005: NGOs and Organizational Change: Discourse, Reporting, and Learning, Cambridge.

ECHO 2004: Generic Security Guide for Humanitarian Organisations, in: http://ec.europa.eu, 27.07.2013.

Edwards, Michael 1997: Becoming a Learning Organization, or, the Search for the Holy Grail?, in: Gillies, David W. (Hrsg.): Strategies of public engagement: Shaping a Canadian Agenda for International Co-Operation, Montreal, 63-94.

EISF 2012: About Us, in: http://www.eisf.eu/about/, 10.08.2012.

EISF 2014a: Members, in: http://www.eisf.eu/about/members.asp, 09.04.2014.

EISF 2014b: Secretariat, in: http://www.eisf.eu/about/staff.asp, 09.04.2014.

Faleg, Giovanni 2012: Between Knowledge and Power: Epistemic Communities and the Emergence of Security Sector Reform in the EU Security Architecture, in: European Security, 21: 2, 161-184.

Fast, Larissa 2010: Mind the gap: Documenting and Explaining Violence Against Aid Workers, in: European Journal of International Relations, 16: 1, 365-389.

Fast, Larissa/O'Neill, Michael 2010: A Closer Look at Acceptance, in: Humanitarian Exchange No. 47, 4-6.

Fast, Larissa/Freeman, C. Faith/O'Neill, Michael/Rowley, Elizabeth 2013: In Acceptance We Trust?: Conceptualising Acceptance as a Viable Approach to NGO Security Management. in: Disasters, 37: 2, 222-243. 
Fearon, James D. 2008: The Rise of Emergency Aid, in: Barnett, Michael/Weiss, Thomas G. (Hrsg.): Humanitarianism in Question: Politics, Power, Ethics, Ithaca, NY, 49-72.

Fearon, James D./Laitin, David D. 2000: Violence and the Social Construction of Ethnic Identity, in: International Organization 54: 4, 845-877.

Finucane, Christopher 2013a: The Cost of Security Risk Management for NGOs. EISF Report, London, in: http://www.eisf.eu/resources/item/?d=7488, 23.07.2013.

Finucane, Christopher 2013b: Risk Management Expense Portfolio Tool, in: http:// www.eisf.eu/resources/item/?d=7485, 23.07.2013.

Froschauer, Ulrike/Lüger, Manfred 2003: Das qualitative Interview. Zur Praxis interpretativer Analyse sozialer Systeme, Stuttgart.

Galbreath, David J./McEvoy, Joanne 2013: How Epistemic Communities Drive International Regimes: The Case of Minority Rights in Europe, in: Journal of European Integration 35: 2, 169-186.

Gallien, Pierre 1998: Project for a humanitarian security network, in: Relief and Rehabilitation Network Newsletter no. 10, February, London, 17.

Gaul, Alexis/Keegan, Michael/Lawrence, Marjorie/Ramos, Maria L. 2006: NGO Security: Does Gender Matter?, in: http://acceptanceresearch.files.wordpress.com/2010/10/ngosecurity-does-gender-matter.pdf, 27.07.2013.

Gebauer, Thomas 2002: Zwischen Befriedung und Eskalation. Zur Rolle der Hilfsorganisationen in Bürgerkriegsökonomien, in: medico international (Hrsg.): Ungeheuer ist nur das Normale. Zur Ökonomie der »neuen« Kriege, Frankfurt/Main, 50-61.

Glaser, Max 2011: Engaging Private Security Providers: A Guideline for Non-Governmental Organisations, London.

Graz, Jean-Christophe/Nölke, Andreas 2008: Introduction: Beyond the fragmented debate on transnational private governance, in: Granz, Jean-Christophe/Nölke, Andreas (Hrsg.): Transnational Private Governance and its Limits, London, 1-26.

Haas, Peter M. 1992a: Introduction: Epistemic Communities and International Policy Coordination, in: International Organization, 46: 1, 1-35.

Haas, Peter M. 1992b: Banning Chlorofluorocarbons: Epistemic Community Efforts to Protect Stratospheric Ozone, in: International Organization, 46: 1, 187-224.

Hall, Rodney B./Biersteker, Thomas J. 2002: The Emergence of Private Authority in the International System, in: Hall, Rodney B./Biersteker, Thomas J. (Hrsg.): The Emergency of Private Authority in Global Governance, Cambridge, 3-22.

Harvey, Paul/Stoddard, Abby/Harmer, Adele/Taylor, Glyn/DiDomenico, Victoria/Brander, Lauren 2010: The State of the Humanitarian System: Assessing Performance and Progress: A Pilot Study, in: http://www.alnap.org/pool/files/alnap-sohs-final.pdf, 23.07.2013.

Hermann, Margaret G. 2009: Content Analysis, in: Klotz, Audie/Prakash, Deepa (Hrsg.): Qualitative Methods in International Relations. A Pluralist Guide, Basingstoke, 151-167.

Humanitarian Outcomes 2012: Aid Worker Security Report 2012, Preview: Figures at a Glance, in: https://aidworkersecurity.org/sites/default/files/AWSDReport2012Preview.pdf, 09.04.2014.

Humanitarian Policy 2013: The Price of Anything, in: http://www.humanitarianpolicy.org/ index.html, 23.07.2013.

Humanitarian Practice Network 2010a: Humanitarian Exchange Number 47, Juni 2010, London.

Humanitarian Practice Network 2010b: Operational Security Management in Violent Environments, Good Practice Review 8, Revised edition, Overseas Development Institute.

ICRC 2007: Stay Safe. The International Federation's Guide for Security Managers, Geneva.

IFRC 2013: Code of Conduct for The International Red Cross and Red Crescent Movement and NGOs in Disaster Relief, in: http://www.ifrc.org/en/publications-and-reports/codeof-conduct, 09.08.2013. 
Ikenberry, John G. 1992: A World Economy Restored: Expert Consensus and the AngloAmerican Postwar Settlement, in: International Organization 46: 1, 289-321.

INSSA 2012a: Programs, in: http://ingossa.org/page/programs, 09.08.2012.

INSSA 2012b: About, http://ingossa.org/page/about, 09.08.2012.

INSSA 2012c: INSSA Code of Conduct, http://ingossa.org/page/codes-of-conduct, 09.08.2012.

INSSA 2013a: About INSSA, in: http://ingossa.org/page/about, 22.07.2013.

INSSA 2013b: Standardization, in: http://ingossa.org/page/standardizations, 22.07.2013.

INSSA 2013c: http://ingossa.org/, 27.07.2013.

InterAction 2006: InterAction Minimum Operating Security Standards, in: http://www.eisf.eu/ resources/item/?d=1612, 23.07.2013.

InterAction 2013: NGO Security, in: http://www.interaction.org/work/security, 11.07.2013.

Irish Aid 2013: Irish Aid Guidelines for NGO Professional Safety \& Security Risk Management, in: http://www.dci.gov.ie/media/irishaid/allwebsitemedia/20newsandpublications/ irish-aid-guidelines-for-ngo-professional-safety-and-security-risk-management.pdf, 09.08.2013.

Kaina, Viktoria 2011: Why Do We Trust Strangers? Revising the Institutional Approach to Generalised Trust Creation, in: West European Politics, 34:2, 289-295.

Keohane, Robert 1984. After Hegemony. Cooperation and Discord in the World Political Economy. Princeton, NJ.

Keck, Margaret E./Sikkink Kathryn 1998: Activists Beyond Borders. Advocacy Networks in International Politics, Ithaca, NY.

Kemp, Edward/Merkelbach, Maarten 2011: Can You Get Sued?: Legal Liability of International Humanitarian Aid Organisations Towards Their Staff, in: http://securitymanagementinitiative.org/index.php?opti-

on $=$ com_docman\&task $=$ cat_view\&gid=22\&Itemid=32\&lang $=$ fr, 27.07.2013.

Levi, Margaret/Murphy, Gillian H. 2006: Coalitions of Contention: The Case of the WTO Protests in Seattle, in: Political Studies, 54: 4, 651-670.

Lieser, Jürgen 2007: Zwischen Macht und Moral. Humanitäre Hilfe der Nichtregierungsorganisationen, in: Treptow, Rainer (Hrsg.): Katastrophenhilfe und Humanitäre Hilfe, München, $40-56$.

Macrae, Joanna 2002: Analysis and Synthesis, in: Macrae, Joanna (Hrsg.): The new humanitarianisms: A Review of Global Trends in Global Humanitarian Action, London, 5-17.

Martin, Randolph 1999: NGO Field Security, in: Forced Migration, 2: 4, 4-7.

Macpherson, Robert 2004: CARE International Safety \& Security Handbook, Atlanta, GA.

Mayring, Philipp 2002: Einführung in die qualitative Sozialforschung. Eine Anleitung zu qualitativem Denken, 5. Auflage. Edition, Weinheim und Basel.

McAdam, Doug/McCarthy, John D./Zald, Mayer N. 1996: Introduction: Opportunities, Mobilizing Structures, and Framing Processes - Toward a Synthetic, Comparative Perspective on Social Movements, in: McAdam, Doug/McCarthy, John D./Zald, Mayer N. (Hrsg.): Comparative Perspectives on Social Movements, Cambridge, MA, 1-20.

Merkelbach, Maarten/Daudin, Pascal 2011: From Security Management to Risk Management. Critical Reflections on Aid Agency Security Management and the ISO Risk Management Guidelines, Geneva.

Micheni, Kiruja 2006: Why Aid Agencies must Strengthen Security Cooperation, in: Ricci, Andrea/Kytömaa, Eero (Hrsg.): Faster and More United? The Debate About Europe's Crisis Response Capacity. Luxembourg, 362-365.

Micheni, Kiruja/Kuhanendran, Janakin 2010: Saving Lives Together: A Review of Security Collaboration Between the United Nations and Humanitarian Actors on the Ground, in: http://www.christianaid.org.uk/images/savinglivestogether.pdf, 24.08.2012.

Mujawar, Shayan 2009: Security Management in Humanitarian Agencies, in: http:// www.ngovoice.org/documents/IMC\%20-\%20Security\%20Management $\% 20 \mathrm{in} \% 20 \mathrm{Hu}-$ manitarian\%20Agencies_Survey\%20Summary.pdf, 19.04.2012. 
Nölke, Andreas 2004: Transnational Private Authority and Corporate Governance, in: Schirm, Stefan (Hrsg.): New Rules for Global Markets: Public and Private Governance in the World Economy. Basingstoke, 155-175.

Peterson, M.J. 1992: Whalers, Cetologists, Environmentalists, and the International Management of Whaling, in: International Organization, 46: 1, 147-186.

PakSafe 2012: About PakSafe, in: http://pakhumanitarianforum.org/paksafe/about-paksafe, 15.03.2012.

Pappi, Franz Urban 1993: Policy-Netze: Erscheinungsform moderner Politiksteuerung oder methodischer Ansatz? in: Héritier, Adrienne (Hrsg.): Policy-Analyse. Kritik und Neuorientierung, Opladen, 84-94.

Pattberg, Philipp 2004: Private-Private Partnerships als innovative Modelle zur Regel(durch)setzung?, in: Brühl, Tanja/Feldt, Heidi/Hamm, Brigitte/Hummel, Hartwig/ Martens, Jens (Hrsg.): Unternehmen in der Weltpolitik. Politiknetzwerke, Unternehmensregeln und die Zukunft des Multilateralismus. Bonn, 143-162.

Persaud, Christine 2012: Gender and Security: Guidelines for Mainstreaming Gender in Security Risk Management. EISF Briefing Paper, London.

PHAP 2013: Mission and Objectives, in: http://phap.org/about-phap/mission-and-objectives, 12.07.2013.

Rathbun, Brian C. 2011a: Before Hegemony: Generalized Trust and the Creation and Design of International Security Organizations, in: International Organization, 65:2, 243-273.

Rathbun, Brian C. 2011 b: From Vicious to Virtuous Circle: Moralistic Trust, Diffuse Reciprocity, and the American Security Commitment to Europe, in: European Journal of International Relations, 18: 2, 323-344.

Radtke, Katrin 2008: Zusammenarbeit mit militärischen Streitkräften, in: http://www.welthungerhilfe.de/fileadmin/media/pdf/Informationsmaterialien/Standpunkt_Nr. 1_2008.pdf, 04.07.2011.

Renouf, Jean 2011: Understanding How the Identity of International Aid Agencies and Their Approaches to Security Are Mutually Shaped: A Thesis Submitted to the Department of International Relations for the Degree of Doctor of Philosophy, in: http://etheses.1se.ac.uk/171/1/Renouf_Understanding_How_the_Identity_of_International_Aid_Agencies_and_Their_Approaches_to_Security_Are_Mutually_Shaped.pdf, $24 . \overline{0} 8.2 \overline{012}$.

Rein, Martin/Schön, Donald 1991: Frame-Reflective Policy Discourse, in: Peter Wagner/ Hirschon Weiss, Carol/Wittrock, Bjorn/Wollmann, Hellmut (Hrsg.): Social Sciences and Modern States: National Experiences and Theoretical Crossroads, Cambridge, MA, 262-289,

Ritzer, George 1975: Professionalization, Bureaucratization and Rationalization: The Views of Max Weber, in: Social Forces 53: 4, 627-634.

Runge, Peter 2009: The Provincial Reconstruction Teams in Afghanistan: Role Model for Civil-Military Relations?, Bonn.

Saab, David J./Taipa, Andrea/Maitland, Carleen/Maldonado, Edgar/ Ngamassi Tchouakeu, Louis-Marie 2013: Inter-organizational Coordination in the Wild: Trust Building and Collaboration Among Field-Level ICT Workers in Humanitarian Relief Organizations, Voluntas, in: International Journal of Voluntary and Nonprofit Organizations 24: 1, 194-213.

Saving Lives Together 2011a: Record of the Second SLT Conference on Security Collaboration Between the United Nations and INGO, in: https://dss.un.org/dssweb/LinkClick.aspx? fileticket=xZxBVMCuXCE\%3d\&tabid=1017\&language=en-US, $\quad 02.08$. 2012.

Saving Lives Together 2011b: Record of the Second Saving Lives Together Conference, in: https://dss.un.org/dssweb/LinkClick.aspx?fileticket=dYnwH9YJb80\%3D\&tabid=1074\&language $=$ en-US, 02.08.2012 
Seabrooke, Leonard/Tsingou, Eleni 2009: Revolving Doors and Linked Ecologies in the World Economy: Policy Locations and the Practice of International Financial Reform, in: http://wrap.warwick.ac.uk/1849/1/WRAP_Seabrooke_26009.pdf, 25.07.2013.

Security Magazine 2011: Most Influential People in Security - 2011, in: http://www.securitymagazine.com/articles/82231-most-influential-people-in-security-2011, 23.07.2013.

Security Magazine 2012: Most Influential People in Security - 2012, in: http://www.securitymagazine.com/articles/83341-the-most-influential-people-in-security-2012, 23.07.2013.

Sending, Ole Jacob/Neumann, Iver B. 2006: Governance and Governmentality: Analyzing NGOs, States, and Power, in: International Studies Quarterly, 50: 3, 651-(3), 651-672.

Seybolt, Taylor B. 2009: Harmonizing the Humanitarian Aid Network: Adaptive Change in a Complex System, in: International Studies Quarterly, 53: 4, 1027-1050.

Smillie, Ian/Minear, Larry 2004: The Charity of Nations. Humanitarian Action in a Calculating World, Bloomfield, CT.

Somalia NGO Consortium 2012: The NGO Safety Programme, in: http://www.somaliangoconsortium.org/services/nsp, 24.02.2012.

Spearin, Christopher 2008: Private, Armed and Humanitarian? States, NGOs, International Private Security Companies and Shifting Humanitarianism, in: Security Dialogue, 39: 4, 363-382.

SPHERE 2011: The Sphere Handbook. Humanitarian Charter and Minimum Standards in Humanitarian Response, in: http://www.sphereproject.org/resources/download-publications/?search $=1 \&$ keywords=\&language $=$ English\&category $=22,27.07 .2013$.

Stephenson, Max Jr. 2005: Making Humanitarian Relief Networks More Effective: Operational Coordination, Trust and Sense Making, in: Disasters, 29: 4, 337-350.

Stoddard, Abby/Harmer, Adele 2010: Supporting Security for Humanitarian Action: A Review of Critical Issues for the Humanitarian Community, London.

Stoddard, Abby/Harmer, Adele/Haver, Katherine 2006: Providing Aid in Insecure Environments: Trends in Policy and Operations, London.

Stoddard, Abby/Harmer, Adele/DiDomenico, Victoria 2009: Providing Aid in Insecure Environments: 2009 Update. Trends in violence against aid workers and the operational response, London.

Stoddard, Abby/Harmer, Adele/Haver, Katherine 2011: Aid Worker Security Report 2011: Spotlight on Security for National Aid Workers: Issues and perspectives, London.

Stoddard, Abby/Harmer, Adele/Hughes, Morgan 2012: Aid Worker Security Report 2012: Host states and their impact on security for humanitarian operations, London.

Suzuki, Naoki 1998: Inside NGOs: Managing Conflicts Between Headquarters and the Field Offices in Non-Governmental Organizations, London.

Торс̧и, Yasemin 1999: Humanitarian NGO-Networks: Identifying Powerful Political Actors in an International Policy-field., in: http://skylla.wz-berlin.de/pdf/1999/p99-302.pdf, 23.05.2005.

Torrente, Nicolas de 2004: Humanitarian Action Under Attack: Reflections on the Iraq War, in: Harvard Human Rights Journal, 17, 1-29.

UN 1999: Humanitarian Assistance and Assistance to Refugees, in: http://www.un.org/ha/ general.htm, 31.03.2010.

Van Brabant, Koenraad 2000: Operational Security Management in Violent Environments: Humanitarian Practice Network, London.

Van Brabant, Koenraad 2001a: Organizational \& Institutional Learning in the Humanitarian Sector, in: Barrow, Ondine/Jennings, Michael (Hrsg.): The Charitable Impulse. NGOs \& Development in East \& North-East Africa, Oxford, 183-199.

Van Brabant, Koenraad 2001b: Mainstreaming the Organisational Management of Safety and Security. A review of aid agency practices and a guide for management: HPG Report No. 9, London.

Van Brabant, Koenraad 2010: Managing Aid Agency Security in an Evolving World: The Larger Challenge, London. 
Vaughn, Jocelyn 2009: The Unlikely Securitizer: Humanitarian Organizations and the Securitization of Indistictiveness, in: Security Dialogue, 40: 3, 263-285.

Weber, Max 2005: Wirtschaft und Gesellschaft. Grundriss der verstehenden Soziologie. Frankfurt/Main.

Wenger, Etienne/McDermott, Richard/Snyder, William M. 2002: A Guide to Managing Knowledge. Cultivating Communities of Practice, Boston, MA.

White, Jim 1997: The International Committee to Protect Aid Workers, in: Relief and Rehabilitation Network Newsletter No. 9, November, London, 17.

Wille, Christina/Fast, Larissa 2013a: Humanitarian Staff Security in Armed Conflict. Insecurity Insight Policy Brief.

Wille, Christina/Fast, Larissa 2013b: Operating in Insecurity. Shifting Patterns of Violence Against Humanitarian Aid Providers and Their Staff (1996-2010), in: Insecurity Insight Report 13-1.

Witzel, Andreas 2000: Das problemzentrierte Interview, in: Forum: Qualitative Sozialforschung, $1: 1$, in: http://www.qualitative-research.net/index.php/fqs/article/view/1132/ $2519 . \% 5 \mathrm{D}, 09.04 .2014$.

Wong, Wendy 2012: Becoming a Household Name: How Human Rights NGOs Establish Credibility Through Organizational Structure, in: Gourevitch, Peter A./Lake, David A./ Gross Stein, Janice (Hrsg.): The Credibility of Transnational NGOs: When Virtue Is Not Enough. Cambridge, 86-111. 